\title{
Arbitrage and utility maximization in market models with an insider*
}

\author{
Huy N. Chau ${ }^{1}$, Wolfgang J. Runggaldier ${ }^{2}$, and Peter Tankov ${ }^{3}$ \\ ${ }^{1}$ Alfréd Rényi Institute of Mathematics, Hungarian Academy of \\ Sciences, Budapest \\ ${ }^{2}$ University of Padova \\ ${ }^{3}$ LPMA, Université Paris-Diderot, corresponding author. E-mail: \\ tankov@math.univ-paris-diderot.fr
}

\begin{abstract}
We study arbitrage opportunities, market viability and utility maximization in market models with an insider. Assuming that an economic agent possesses an additional information in the form of an $\mathscr{F}_{T}$-measurable random variable $G$, we give criteria for the No Unbounded Profits with Bounded Risk property to hold, characterize optimal arbitrage strategies, and prove duality results for the utility maximization problem faced by the insider. Examples of markets satisfying NUPBR yet admitting arbitrage opportunities are provided for both atomic and continuous random variables $G$.
\end{abstract}

Key words: Initial enlargement of filtration, optimal arbitrage, No Unbounded Profits with Bounded Risk, incomplete markets, hedging, utility maximization.

JEL Classification: G14

\section{Introduction}

The aim of this paper is to study arbitrage opportunities and utility maximization in market modes with an insider. Insider information is typically modeled by using the mathematical theory of enlargement of filtration, where one distinguishes initial, successive and progressive enlargement. In this paper we restrict ourselves to the setting of initial enlargement by a random variable $G$ : at time zero the insider knows the realization of $G$, which the ordinary agents only observe at the end of the trading period that we shall assume to be finite. Note that some concepts of arbitrage under initial enlargement and progressive enlargement on an infinite horizon have recently been studied in [1] and [2].

Insider trading under initial enlargement of filtration has been the object of interest of many papers, including but by no means limited to [12, 3, 16, 6, 7, 4, 32, 17]. The majority of these papers work in a complete market setting and are concerned with

\footnotetext{
${ }^{*}$ The research of Chau Ngoc Huy was supported by Natixis Foundation for Quantitative Research and the "Lendület" grant LP2015-6 of the Hungarian Academy of Sciences. The research of Peter Tankov was supported by the chair "Financial Risks" sponsored by Société Générale.
} 
the question of additional utility of the insider; they find that when the variable $G$ is $\mathscr{F}_{T}$-measurable and not purely atomic, this additional utility is often infinite.

In contrast to these papers, our main interest lies in exploring various concepts of arbitrage in the context of initial filtration enlargement. In particular, we are interested in the following questions.

- When does the market for the insider satisfy the property NUPBR (no unbounded profit with bounded risk)? The NUPBR condition, see [24], or, equivalently, No Arbitrage of the first kind (NA1) (see also NAA1 in [23], and BK in [22]), boils down to assuming that no positive claim, which is not identically zero, may be superhedged at zero price. It is the minimal condition enabling one to solve portfolio optimization problems in a meaningful way: in [24] it is shown that, without NUPBR, one has either no solution or infinitely many. NUPBR is robust with respect to changes of numeraire, absolutely continuous measure change and, in some cases, change of reference filtration (see e.g. [15]). Finally, it is also known [24] that NFLVR (the classical assumption of no free lunch with vanishing risk) is equivalent to NUPBR plus the classical no arbitrage assumption (NA), which means that markets with NUPBR can still admit (unscalable) arbitrage opportunities.

- When does the market for the insider admit optimal arbitrage? We say that a financial market admits optimal arbitrage if there exists a strategy which allows to superhedge a unit amount with an initial cost which is strictly less than one in some states of nature (note that the initial cost of a strategy for the insider may be a random variable since the insider possesses a nontrivial information already at time $t=0$ ). We say that the optimal arbitrage is strong whenever the replication cost is strictly less than one with probability one. In other words, an optimal arbitrage strategy allows to replicate a risk-free zero coupon bond at a price which is strictly less than the initial price of this bond (see [8] for possible uses of such strategies in the context of asset liability management for e.g., pension funds).

To address the above questions, we distinguish the cases when the additional information is represented by a discrete (atomic) random variable $G$ and when it is given by a random variable $G$ which is not purely atomic. The discrete case is the easier one, and allows us to provide full answers to the above questions. Namely, the following results are shown to be true under natural assumptions in the case when $G$ is discrete.

- The market for the insider satisfies the NUPBR property.

- If the original market (for non-informed agents) is complete, then the market for the insider admits strong optimal arbitrage. If the original market is incomplete, the optimal arbitrage may or may not exist, and we give examples of both situations.

The case when $G$ is not purely atomic is more difficult, and only partial answers to the above questions are provided in this paper for this case. Our first contribution here is to establish a new necessary condition for the insider market to satisfy the NUPBR property. This condition is, in particular, violated by all complete markets, which means that complete markets always admit an arbitrage of the first kind. In the incomplete markets the situation is less clear, and we provide examples of both an incomplete market violating NUPBR and of an incomplete market for which NUPBR 
holds and logarithmic utility of the insider is finite, although the market admits arbitrage opportunities.

In addition to the above results, we also address the problem of utility optimization for the insider. In this context, our contribution is two-fold. First, we show that the utility maximization problem for the insider may be expressed in terms of the quantities (strategies, martingale measures) defined in the original market for the uninformed agents. This in turn allows us to develop an extension of the classical duality results for utility maximization to market models with an insider. These results are first obtained in the case of a discrete initial information $G$, and then extended to a non purely atomic $G$ with a limiting procedure.

The rest of the paper is structured as follows. In section 2 we introduce the market model and recall the basic notions of no-arbitrage and filtration enlargement.

In section 3 we deal with initial enlargement by a discrete random variable $G$. We show that, under a suitable assumption, a market initially enlarged with a discrete r.v. $G$ always satisfies NUPBR. In subsection 3.1 we then study optimal arbitrage that can be implemented via superhedging. We show that the superhedging price of a given claim for the insider may be represented in terms of the superhedging prices in the filtration of ordinary agents of the claim restricted to the events corresponding to the various possible values of $G$. In subsection 3.2 we consider portfolio optimization, in particular the maximization of expected utility and obtain a duality relationship. An example computation of optimal arbitrage and maximal expected log-utility for the insider in an incomplete market is presented in subsection 3.3 .

In section 4we study the initial enlargement with a random variable $G$, which is not purely atomic. We first show that if the set of possible martingale densities is uniformly integrable, then NUPBR cannot hold. We then present an approximation procedure allowing to obtain results for a general random variable $G$ by a limiting procedure from the results obtained for a discrete variable $G$ in section 3 . This procedure allows us to extend the results on utility optimization to the case of general $G$ in subsection 4.3 Finally, the Appendix contains some technical proofs.

\section{Market model and preliminaries related to filtration enlargement}

In this section we introduce our basic market model and recall known concepts as we shall use them in the sequel (subsection 2.1). We then introduce some preliminaries in relation to filtration enlargement (subsection 2.2).

\subsection{Market model and basic notions}

On a stochastic basis $(\Omega, \mathscr{F}, \mathbb{F}, \mathbb{P})$, where the filtration $\mathbb{F}=\left(\mathscr{F}_{t}\right)_{t \geq 0}, t \leq T$ satisfies the usual conditions, consider a financial market with an $\mathbb{R}^{d}$-valued nonnegative semimartingale process $S=\left(S^{1}, \ldots, S^{d}\right), t \leq T$, where the components represent the prices of $d$ risky assets. The horizon is supposed to be finite and given by $T>0$. We assume that the price processes are already discounted, namely for the riskless asset price $S^{0}$ we assume $S^{0} \equiv 1$, and that this market is frictionless. Let $L(S)$ be the set of all $\mathbb{R}^{d}$-valued $S$-integrable predictable processes and, for $H \in L(S)$ denote by $H \cdot S$ the vector stochastic integral of $H$ with respect to $S$. 
Definition 2.1. An investment strategy $H$ is an element $H \in L(S)$, where the components indicate the number of units invested in the individual assets. Letting $x \in \mathbb{R}_{+}$, an $H \in L(S)$ is said to be an $x$-admissible strategy, if $H_{0}=0$ and $(H \cdot S)_{t} \geq-x$ for all $t \in[0, T], \mathbb{P}$-a.s. $H \in L(S)$ is said to be admissible if it is $x$-admissible for some $x \in \mathbb{R}_{+}$. We denote by $\mathscr{A}_{x}$ the set of all $x$-admissible strategies and by $\mathscr{A}$ that of all admissible strategies. For $(x, H) \in \mathbb{R}_{+} \times \mathscr{A}$ we define the portfolio value process $V_{t}^{x, H}:=x+(H \cdot S)_{t}$ implying that $x$ is the amount of the initial wealth and that portfolios are generated only by self-financing admissible strategies. Finally, we denote by $\mathscr{K}_{x}$ the set of all claims that one can realize by $x$-admissible strategies starting with zero initial cost:

$$
\mathscr{K}_{x}=\left\{V_{T}^{0, H} \mid H \in \mathscr{A}_{x}\right\}
$$

and $\mathscr{K}$ denotes the set of claims that can be replicated with zero initial cost and any admissible strategy: $\mathscr{K}=\cup_{x \geq 0} \mathscr{K}_{x}$.

Let

$$
\mathscr{C}=\left(\mathscr{K}-L_{+}^{0}\right) \cap L^{\infty} .
$$

In the sequel, we shall use the following no-arbitrage conditions.

Definition 2.2 (NFLVR). We say that there is No Free Lunch with Vanishing Risk if

$$
\overline{\mathscr{C}} \cap L_{+}^{\infty}=\{0\}
$$

where the closure is taken with respect to the topology of uniform convergence.

Definition 2.3 (NUPBR). There is No Unbounded Profit With Bounded Risk if the set $\mathscr{K}_{1}$ is bounded in $L^{0}$, that is, if

$$
\lim _{c \uparrow \infty} \sup _{W \in \mathscr{K}_{1}} P(W>c)=0
$$

The NUPBR condition can be shown to be equivalent to the following, more economically meaningful condition, which boils down to assuming that no positive claim, which is not identically zero, may be superhedged at zero price with a positive portfolio (see [21] for a recent discussion of the different equivalent formulations of NUPBR).

Definition 2.4 (NA1). An $\mathscr{F}_{T}$-measurable random variable $\xi$ is called an Arbitrage of the First Kind if $\mathbb{P}(\xi \geq 0)=1, \mathbb{P}(\xi>0)>0$, and for all $x>0$ there exists an admissible strategy $H \in \mathscr{A}_{x}$ such that $V_{T}^{x, H} \geq \xi$. We shall say that the market admits No Arbitrage of the First Kind (NA1), if no such random variable exists.

Definition 2.5 (Classical arbitrage). We shall say that $H \in \mathscr{A}$ is an arbitrage strategy if $P\left(V_{T}^{0, H} \geq 0\right)=1$ and $\mathbb{P}\left(V_{T}^{0, H}>0\right)>0$. It is a strong arbitrage if $\mathbb{P}\left(V_{T}^{0, H}>0\right)=1$. An arbitrage strategy is said to be scalable if $H \in \mathscr{A}_{0}$ and unscalable if $H \in \mathscr{A}_{x}$ with $x>0$ for some $x$, but $H \notin \mathscr{A}_{0}$. We shall say that there is absence of classical arbitrage, denoted by NA, if there are no scalable or unscalable arbitrage strategies, that is,

$$
\mathscr{C} \cap L_{+}^{\infty}=\{0\} .
$$

In our context (nonnegative processes), NFLVR is equivalent to the existence of at least one equivalent local martingale measure [13]. The set of all such measures will be denoted by $\operatorname{ELMM}(\mathbb{F}, \mathbb{P})$ and the set of corresponding densities will be denoted by $\operatorname{ELMMD}(\mathbb{F}, \mathbb{P})$. NUPBR is, in turn, equivalent to the existence of a local martingale deflator [25, 37, 36]. In addition, NFLVR is equivalent to NUPBR plus NA [24]. 


\subsection{Preliminaries in relation to filtration enlargement}

We start again from a filtered probability space $(\Omega, \mathscr{F}, \mathbb{F}, \mathbb{P})$, on which we consider a financial market with an insider.

Assumption 2.6. The $(\mathbb{F}, \mathbb{P})$-market of regular agents satisfies NFLVR implying that the set $E L M M D(\mathbb{F}, \mathbb{P})$ is not empty. The insider possesses from the beginning an additional information about the outcome of some $\mathscr{F}_{T}-$ measurable r.v. $G$ with values in $(\mathbb{R}, \mathscr{B})$.

Starting from $\mathbb{F}=\left(\mathscr{F}_{t}\right)$ one can then consider the (initially) enlarged filtration $\mathbb{G}=$ $\left(\mathscr{G}_{t}\right)$ with

$$
\mathscr{G}_{t}=\cap_{\varepsilon>0}\left(\mathscr{F}_{t+\varepsilon} \vee \sigma(G)\right)
$$

In the context of filtration enlargements it is important to have a criterion which ensures that an $\mathbb{F}$-local martingale remains a $\mathbb{G}$-semimartingale. In view of introducing the corresponding condition, let $v_{t}:=\mathbb{P}\left\{G \in d x \mid \mathscr{F}_{t}\right\}$ be the regular conditional distribution of $G$, given $\mathscr{F}_{t}$, and $v:=\mathbb{P}\{G \in d x\}$ be the law of $G$. We shall require Jacod's condition (see [18]) in the following form

Assumption 2.7. (Absolutely continuous version of Jacod's condition). We assume that

$$
v_{t} \ll v, \quad \mathbb{P}-\text { a.s. for } t<T \text {. }
$$

Notice that the absolute continuity is imposed only before the terminal time $T$. In our setting, where $G \in \mathscr{F}_{T}$, the absolute continuity cannot hold at the terminal date. Some papers on insider trading require that $v_{t} \sim v$ (see, e.g., [4]) but this would imply that the density of $v_{t}$ with respect to $v$ is strictly positive and so allow one to construct an equivalent martingale measure from the density process before the terminal time $T$ [4]. This would imply NFLVR for the $(\mathbb{G}, \mathbb{P})$ - market (before time $T$ ) and thus exclude arbitrage possibilities there, which is not our purpose.

We need one more assumption, which refers to the density process of $v_{t}$ with respect to $v$. To this effect we first recall from Lemme 1.8 and Corollaire 1.11 of [18] that we can choose a nice version of the density, namely we have the following lemma where $\mathscr{O}(\mathbb{F})$ denotes the $\mathbb{F}$-optional sigma field on $\Omega \times \mathbb{R}_{+}$.

Lemma 2.8. Under Assumption 2.7 there exists a nonnegative $\mathscr{B} \otimes \mathscr{O}(\mathbb{F})$-measurable function $\mathbb{R} \times \Omega \times \mathbb{R}_{+} \ni(x, \omega, t) \mapsto p_{t}^{x}(\omega) \in[0, \infty)$, càdlàg in $t$ such that

1. for every $t \in[0, T)$, we have $v_{t}(d x)=p_{t}^{x}(\omega) v(d x)$.

2. for each $x \in \mathbb{R}$, the process $\left(p_{t}^{x}(\omega)\right)_{t \in[0, T)}$ is a $(\mathbb{F}, \mathbb{P})$-martingale.

3. The processes $p^{x}, p_{-}^{x}$ are strictly positive on $\left[0, \tau^{x}\right)$ and $p^{x}=0$ on $\left[\tau^{x}, T\right)$, where

$$
\tau^{x}:=\inf \left\{t \geq 0: p_{t-}^{x}=0 \text { or } p_{t}^{x}=0\right\} \wedge T .
$$

Furthermore, if we define $\tau^{G}(\omega):=\tau^{G(\omega)}(\omega)$ then $\mathbb{P}\left[\tau^{G}=T\right]=1$.

The conditional density process $p^{G}$ is also the key to find the semimartingale decomposition of an $\mathbb{F}$-local martingale in the enlarged filtration $\mathbb{G}$.

We come now to the announced additional assumption

Assumption 2.9. For every $x$, the process $p^{x}$ does not jump to zero, i.e.

$$
\mathbb{P}\left[\tau^{x}<T, p_{\tau^{x}-}^{x}>0\right]=0 .
$$


This assumption is used in [26] for a general construction of strict local martingales, in [35] for a construction of markets with arbitrages and in [10] for the study of optimal arbitrage when agents have non equivalent beliefs. This assumption is also used to prove the preservation of NUPBR in the enlarged market over an infinite horizon, see [1] or Theorem 6(a) of [2].

\section{Enlargement with a discrete random variable}

In this section we consider the case when the random variable $G$ of the (initial) enlargement is a discrete random variable $G \in\left\{g_{1}, \ldots, g_{n}\right\}$, with $n \geq 2$ and $\mathbb{P}\left[G=g_{i}\right]>0$ for all $i$. After a general theorem concerning NUPBR for this case, we study optimal arbitrage in subsection 3.1 and provide a dual representation for expected utility maximization in subsection 3.2. An example for computing optimal arbitrage and maximal expected utility for the insider is presented in subsection 3.3 for the case of an incomplete market.

Notice first that the initial enlargement with a discrete random variable is a classical case studied already by P. A. Meyer [30] and by many other authors. For this case it is known that every $\mathbb{F}$-local martingale is a $\mathbb{G}$-semimartingale on $[0, T]$ and it is not necessary to impose Jacod's condition. We shall however make the Assumption 2.9.

In the discrete case the insider can update her belief with a measure change $\mathbb{P} \rightarrow \mathbb{Q}^{i}$ thereby dismissing all scenarios not contained in $\left\{G=g_{i}\right\}$. The measure $\mathbb{Q}^{i}$ satisfies

$$
\left.\frac{d \mathbb{Q}^{i}}{d \mathbb{P}}\right|_{\mathscr{F}_{t}}=\frac{\mathbb{P}\left\{G=g_{i} \mid \mathscr{F}_{t}\right\}}{\mathbb{P}\left\{G=g_{i}\right\}}:=p_{t}^{g_{i}}
$$

It gives total mass to $\left\{G=g_{i}\right\}$ and is absolutely continuous but not equivalent to $P$.

The following theorem shows that NUPBR always holds true in this setting.

Theorem 3.1. Let $G$ be discrete and suppose that Assumptions 2.6 and 2.9 hold true. Then the $(\mathbb{G}, \mathbb{P})$-market satisfies NUPBR.

Proof. The statement is proved by way of contradiction, noticing that NUPBR is equivalent to NA1. Assume that there is an arbitrage of the first kind in the $(\mathbb{G}, \mathbb{P})$-market, i.e., we can find an $\mathscr{F}_{T}$-measurable random variable $\xi$ (because $\mathscr{F}_{T}=\mathscr{G}_{T}$ ) such that $\mathbb{P}[\xi \geq 0]=1, \mathbb{P}[\xi>0]>0$ and for all $\varepsilon>0$, there exists a $\mathbb{G}$-predictable strategy $H^{\mathbb{G}, \varepsilon}$ which satisfies

$$
\varepsilon+\left(H^{\mathbb{G}, \varepsilon} \cdot S\right)_{T} \geq \xi, \mathbb{P}-\text { a.s. }
$$

Choose an index $i$ such that $\mathbb{P}\left[\{\xi>0\} \cap\left\{G=g_{i}\right\}\right]>0$. The inequality (2) still holds true under $\mathbb{Q}^{i}$ in the form of

$$
\varepsilon+\left(H^{\mathbb{G}, \varepsilon} 1_{G=g_{i}} \cdot S\right)_{T} \geq \xi, \mathbb{Q}^{i}-\text { a.s. }
$$

Let us look at the hedging strategy $H^{\mathbb{G}, \varepsilon} 1_{G=g_{i}}$ under $\mathbb{Q}^{i}$. Recall that (see [20]) the predictable process $H^{\mathbb{G}, \varepsilon}$ is of the form $H_{t}^{\mathbb{G}, \varepsilon}(\omega)=h_{t}(\omega, G(\omega))$ where $h_{t}(\omega, x)$ is a $\mathscr{P}(\mathbb{F}) \times \mathscr{B}(\mathbb{R})$ - measurable function with $\mathscr{P}(\mathbb{F})$ denoting the $\mathbb{F}$-predictable $\sigma$-algebra on $\Omega \times \mathbb{R}_{+}$. Then $H^{\mathbb{G}, \varepsilon, i}:=h\left(\omega, g_{i}\right)$ is $\mathbb{F}$-predictable and we have the representation $H^{\mathbb{G}, \varepsilon} \mathbf{1}_{\left\{G=g_{i}\right\}}=\tilde{H}^{\mathbb{F}, \varepsilon, i} \mathbf{1}_{\left\{G=g_{i}\right\}}$ where $\tilde{H}^{\mathbb{F}, i, \varepsilon}$ is a $\mathbb{F}$-predictable strategy. Thus (3) implies that $\xi$ is an arbitrage of the first kind in the $\left(\mathbb{F}, \mathbb{Q}^{i}\right)$-market, which is equivalent to the failure of NUPBR in the $\mathbb{Q}^{i}$-market. Notice next that, for each $i \in\{1, \ldots, n\}$, the 
$\left(\mathbb{F}, \mathbb{Q}^{i}\right)$-market is obtained from the $(\mathbb{F}, \mathbb{P})$-market by an absolutely continuous measure change, see (1). Furthermore, the density process $p^{g_{i}}$ does not jump to zero, by Assumption 2.9. By Theorem 4.1 of [10] this implies that the condition NUPBR holds for the $\left(\mathbb{F}, \mathbb{Q}^{i}\right)$-market thus proving the contradiction and with it the statement.

Recently Acciaio et al. [1] gave sufficient conditions for NUPBR to hold in the $(\mathbb{G}, \mathbb{P})$ - market by constructing a martingale deflator under $G$ (see also the introductory part to section 4 below). The construction of local martingale deflators is also given in Proposition 10 (for quasi left-continuous $\mathbb{F}$-local martingales), Proposition 11, and Theorem 6 of [2]. However they work on an infinite horizon requiring the absolute continuity in Jacod's hypothesis to hold at all times and so their approach cannot be adapted to our finite horizon case.

Theorem 3.1 shows that, under the Assumption 2.9. the $(\mathbb{G}, \mathbb{P})$-market satisfies NUPBR; it does not exclude that it satisfies also NFLVR. This depends on the possibility of classical arbitrage in the various specific cases. The study of such arbitrage opportunities is the subject of the next section.

\subsection{Optimal arbitrage via superhedging}

The notion of optimal arbitrage goes back to [14]. Here, following [10], we relate optimal arbitrage to superhedging. We start from a definition of the superhedging price which is adapted to the context of filtration enlargement. Whenever in the sequel the filtration may be either $\mathbb{F}$ of $\mathbb{G}$, we shall use the symbol $\mathbb{H} \in\{\mathbb{F}, \mathbb{G}\}$.

Definition 3.2. Let $\mathbb{H} \in\{\mathbb{F}, \mathbb{G}\}$ and let $f \geq 0$ be a given claim. An $\mathscr{H}_{0}$-measurable random variable $x_{*}^{\mathbb{H}}(f)$ is called the superhedging price of $f$ with respect to $\mathbb{H}$ if there exists an $\mathbb{H}$-predictable strategy $H$ such that

$$
\left\{\begin{array}{lll}
x_{*}^{\mathbb{H}}(f)+(H \cdot S)_{t} & \geq 0, & \mathbb{P}-a . s, \forall t \in[0, T], \\
x_{*}^{\mathbb{H}}(f)+(H \cdot S)_{T} & \geq f, & \mathbb{P}-\text { a.s. }
\end{array}\right.
$$

and, if any $x \in \mathscr{H}_{0}$ satisfies these conditions, then $x_{*}^{\mathbb{H}}(f) \leq x, \mathbb{P}-$ a.s.

In other words, the superhedging price of $f$ is the essential lower bound of the initial values of all nonnegative admissible portfolio processes, whose terminal value dominates $f$. Notice that $\mathscr{G}_{0}$ is non trivial implying that the superhedging price $x_{*}^{\mathbb{G}}(f)$ is a random variable. However, this price is constant on each event $\left\{G=g_{i}\right\}$.

We come next to the superhedging theorem that shows how the superhedging price and a superhedging strategy for $f$ in $\mathbb{G}$ can be obtained in terms of the superhedging price and the associated strategy in $\mathbb{F}$ by restricting $f$ to the individual events $\left\{G=g_{i}\right\}$.

Theorem 3.3. Let $G$ be discrete and suppose that Assumptions 2.6 and 2.9 hold true. Then,

i) The superhedging price for a claim $f \geq 0$ in the $(\mathbb{G}, \mathbb{P})$-market is given by

$$
x_{*}^{\mathbb{G}}(f)=\sum_{i=1}^{n} x_{*}^{\mathbb{F}}\left(f \mathbf{1}_{\left\{G=g_{i}\right\}}\right) \mathbf{1}_{\left\{G=g_{i}\right\}}
$$

ii) The associated hedging strategy is $\sum_{i=1}^{n} H^{\mathbb{F}, i} \mathbf{1}_{\left\{G=g_{i}\right\}}$ where $H^{\mathbb{F}, i}$ is the superhedging strategy for $f \mathbf{1}_{\left\{G=g_{i}\right\}}$ in the $(\mathbb{F}, \mathbb{P})$-market, i.e.

$$
\sum_{i=1}^{n} x_{*}^{\mathbb{F}}\left(f \mathbf{1}_{\left\{G=g_{i}\right\}}\right) \mathbf{1}_{\left\{G=g_{i}\right\}}+\sum_{i=1}^{n}\left(H^{\mathbb{F}, i} \mathbf{1}_{\left\{G=g_{i}\right\}} \cdot S\right)_{T} \geq f, \mathbb{P}-\text { a.s. }
$$


Remark 3.4. Using this theorem, the computation of the superhedging price for the insider reduces to the computation of the superhedging price for the uninformed agents in the original market, which satisfies NFLVR. In particular, using the classical superhedging duality, we may write

$$
x_{*}^{\mathbb{G}}(f)=\sum_{i} \sup _{Z \in E L M M D(\mathbb{F}, \mathbb{P})} \mathbb{E}^{\mathbb{P}}\left[Z f \mathbf{1}_{\left\{G=g_{i}\right\}}\right] \mathbf{1}_{\left\{G=g_{i}\right\}} .
$$

Proof. As in the proof of Theorem 3.1 here we also make use of Theorem 4.1 in [10], which relates the superhedging price under a measure $\mathbb{P}$ to that under a measure $\mathbb{Q}$, with respect to which $\mathbb{P}$ is only absolutely continuous, but not necessarily equivalent. The role of the measure $\mathbb{Q}$ in $[10]$ will be played here by the measure $\mathbb{P}$ and that of $\mathbb{P}$ in [10] by the various measures $\mathbb{Q}^{i}$ defined in (1). To make clear which measure is being used, in this proof we shall use the notation $x_{*}^{\mathbb{F}, \mathbb{P}}(\cdot)$ or $x_{*}^{\mathbb{F}, \mathbb{Q}^{i}}(\cdot)$ respectively.

Theorem 4.1 of [10] leads to

$$
x_{*}^{\mathbb{F}, \mathbb{Q}^{i}}(f)=x_{*}^{\mathbb{F}, \mathbb{P}}\left(f 1_{G=g_{i}}\right) .
$$

For each $i$, we denote by $H^{\mathbb{F}, i}$ the $\mathbb{F}$-predictable strategy which superhedges $f$ in the $\left(\mathbb{F}, \mathbb{Q}^{i}\right)$-market, that is

$$
x_{*}^{\mathbb{F}, \mathbb{Q}^{i}}(f)+\left(H^{\mathbb{F}, i} \cdot S\right)_{T} \geq f, \quad \mathbb{Q}^{i}-\text { a.s. }
$$

This inequality holds also under $\mathbb{P}$ when restricted on $\left\{G=g_{i}\right\}$, namely

$$
x_{*}^{\mathbb{F}, \mathbb{P}}\left(f 1_{G=g_{i}}\right) 1_{G=g_{i}}+\left(H^{\mathbb{F}, i} 1_{G=g_{i}} \cdot S\right)_{T} \geq f 1_{G=g_{i}}, \mathbb{P}-\text { a.s. }
$$

Summing up these inequalities we obtain

$$
\left(\sum_{i} x_{*}^{\mathbb{F}, \mathbb{P}}\left(f 1_{G=g_{i}}\right) 1_{G=g_{i}}\right)+\left(\left(\sum_{i} H^{\mathbb{F}, i} 1_{G=g_{i}}\right) \cdot S\right)_{T} \geq f, \mathbb{P}-\text { a.s. }
$$

The hedging strategy $\left(\sum_{i} H^{\mathbb{F}, i} 1_{G=g_{i}}\right)$ is $\mathbb{G}$-predictable.

Finally, we prove that the initial capital $\sum_{i} x_{*}^{\mathbb{F}, \mathbb{P}}\left(f 1_{G=g_{i}}\right) 1_{G=g_{i}}$ is exactly the superhedging price of $f$ in the $(\mathbb{G}, \mathbb{P})$-market. Assume that $y$ is a $\mathscr{G}_{0}$-measurable random variable such that

$$
y+\left(H^{\mathbb{G}} \cdot S\right)_{T} \geq f, \mathbb{P}-\text { a.s. }
$$

where $H^{\mathbb{G}}$ is a $\mathbb{G}$-predictable strategy. Hence,

$$
y 1_{G=g_{i}}+\left(H^{\mathbb{G}} 1_{G=g_{i}} \cdot S\right)_{T} \geq f 1_{G=g_{i}}, \mathbb{P}-\text { a.s. }
$$

Because $\mathbb{Q}^{i} \ll \mathbb{P}$ with $\mathbb{Q}^{i}\left[G=g_{i}\right]=1$, we obtain

$$
y+\left(H^{\mathbb{G}} 1_{G=g_{i}} \cdot S\right)_{T} \geq f, \mathbb{Q}^{i}-\text { a.s. }
$$

By using the same argument as in the proof of Theorem 3.1 we can replace $H^{\mathbb{G}}$ on the various events $\left\{G=g_{i}\right\}$ by an $\mathbb{F}$-predictable strategy $\tilde{H}^{\mathbb{F}, i}$ and then

$$
y+\left(\tilde{H}^{\mathbb{F}, i} \cdot S\right)_{T} \geq f, \mathbb{Q}^{i}-\text { a.s. }
$$

By definition, the superhedging price of $f$ under $\mathbb{Q}^{i}$ is not greater than $y$ and so we conclude that $\sum_{i} x_{*}^{\mathbb{F}, \mathbb{P}}\left(f 1_{G=g_{i}}\right) 1_{G=g_{i}} \leq y, \mathbb{P}-$ a.s. 
We now give a specific definition of a market with optimal arbitrage which is adapted to the context of initial filtration enlargement and is motivated by Lemma 3.3 in [10].

Definition 3.5. There is optimal arbitrage in the $(\mathbb{H}, \mathbb{P})$-market if $x_{*}^{\mathbb{H}}(1) \leq 1$ and $\mathbb{P}\left\{x_{*}^{\mathbb{H}}(1)<1\right\}>0$. If $x_{*}^{\mathbb{H}}(1)<1, \mathbb{P}-$ a.s. then the optimal arbitrage is said to be strong.

From this definition and Theorem 3.3 it follows that there is optimal arbitrage for the insider if and only if $x_{*}^{\mathbb{F}}\left(\mathbf{1}_{\left\{G=g_{i}\right\}}\right)<1$ for some $i$. If the market is complete, then $x_{*}^{\mathbb{F}}\left(\mathbf{1}_{\left\{G=g_{i}\right\}}\right)=\mathbb{E}^{\mathbb{P}}\left[Z \mathbf{1}_{\left\{G=g_{i}\right\}}\right]<1$ for all $i$, where $Z$ is the density of the unique martingale measure. Therefore, in a complete market there always exists strong optimal arbitrage.

It follows from Remark 3.4 in [10] that, under NUPBR, one has $x_{*}^{\mathbb{H}}(1)>0 \mathbb{P}$-a.s. However, $x_{*}^{\mathbb{H}}(1)>0$ does not imply NUPBR (see [29] for a market model that does not satisfy NUPBR, but satisfies NA, which implies $\left.x_{*}^{\mathbb{H}}(1)>0\right)$.

\subsection{Expected utility maximization for an insider}

This subsection concerns utility maximization. We first formulate the precise relationship between absence of arbitrage, in particular NUPBR, and utility maximization, and then show that expected utility maximization in the enlarged market can be performed by an analog of classical duality also under absence of an ELMMD.

Given a concave and strictly increasing utility function $U(\cdot)$, the corresponding portfolio optimization problem is given by

$$
u(x):=\sup _{H \in \mathscr{A}_{x}} \mathbb{E}\left\{U\left(V_{T}^{x, H}\right)\right\}
$$

Recall that it is shown in [24] that, if NUPBR fails, then $u(x)=+\infty$ for all $x>0$ or the problem has infinitely many solutions. This result implies immediately the following statement, which we formulate as a proposition because of its importance. It holds in general and not only in the specific case of this section.

Proposition 3.6. Assume that the utility function is strictly increasing, concave, and satisfies $U(+\infty)=+\infty$. If there exists $x>0$ for which $u(x)<+\infty$, then NUPBR holds.

In particular, a criterion allowing to show that NUPBR holds is thus to show that e.g. the log-utility maximization leads to a finite value. Notice, however, that NUPBR does not imply that expected utility is finite.

We now discuss the duality approach for utility maximization. Before stating the main theorem, we prove one preliminary lemma. This lemma, which we state for a general increasing function, shows that it is possible to relate the expected utility of the insider to the expected utility of regular agents when restricted to the events $\left\{G=g_{i}\right\}$. In this lemma and below we denote by $\mathscr{A}_{1}^{\mathbb{F}}$ and $\mathscr{A}_{1}^{\mathbb{G}}$ the set of 1 -admissible strategies that are predictable with respect to $\mathbb{F}$ and $\mathbb{G}$ respectively.

Lemma 3.7. Let $U$ be an increasing function. Then,

$$
\sup _{H \in \mathscr{A}_{1}^{\mathbb{G}}} \mathbb{E}^{\mathbb{P}}\left[U\left(V_{T}^{1, H}\right)\right]=\sum_{i=1}^{n} \sup _{H \in \mathscr{A}_{1}^{\mathbb{P}}} \mathbb{E}^{\mathbb{P}}\left[U\left(V_{T}^{1, H}\right) 1_{G=g_{i}}\right]
$$


Proof. The proof only requires the representation of $\mathbb{G}$-predictable processes as it was used in the proofs of Theorems 3.1 and 3.3 . We do not need Assumption 2.9 here.

$(\leq)$ Let $H^{\mathbb{G}} \in \mathscr{A}^{\mathbb{G}}$ be a $\mathbb{G}$-predictable strategy. As mentioned above, the $\mathbb{G}$ predictable process $H^{\mathbb{G}}$ can be expressed as $H_{t}^{\mathbb{G}}(\omega)=h_{t}(\omega, G(\omega))$ where $h_{t}(\omega, x)$ is a $\mathscr{P}(\mathbb{F}) \times \mathscr{B}(\mathbb{R})$-measurable function. Then, $H^{\mathbb{F}, i}=h\left(\omega, g_{i}\right)$ is $\mathbb{F}$-predictable and $H^{\mathbb{G}} 1_{G=g_{i}}=H^{\mathbb{F}, i} 1_{G=g_{i}}$ a.s. Hence, we have that $H^{\mathbb{G}}=\sum_{i=1}^{n} H^{\mathbb{F}, i} 1_{G=g_{i}}$ and therefore

$$
\int_{0}^{T} H_{t}^{\mathbb{G}} d S_{t}=\sum_{i=1}^{n} 1_{G=g_{i}} \int_{0}^{T} H_{t}^{\mathbb{F}, i} d S_{t}
$$

where the equality follows from the fact that $S$ is a $\mathbb{G}$-semimartingale. Consequently

$$
\mathbb{E}^{\mathbb{P}}\left[U\left(V_{T}^{1, H^{\mathbb{G}}}\right)\right]=\sum_{i=1}^{n} \mathbb{E}^{\mathbb{P}}\left[1_{G=g^{i}} U\left(V_{T}^{1, H^{\mathbb{E}, i}}\right)\right],
$$

where we used the fact that $U$ is increasing to take the expectation since it implies that both expressions under the $\mathbb{E}$ sign are bounded from below. Taking the supremum over the set of all $\mathbb{G}$-admissible strategies we obtain the inequality $(\leq)$ in (5).

$(\geq)$ Let $H^{\mathbb{F}, i}, i=1, \ldots, n$ be $\mathbb{F}$-predictable strategies. Then, the strategy $H^{\mathbb{G}}=$ $\sum_{i=1}^{n} H^{\mathbb{F}, i} 1_{G=g_{i}}$ is $\mathbb{G}$-predictable and the following straightforward inequality completes the proof.

$$
\sum_{i=1}^{n} \mathbb{E}^{\mathbb{P}}\left[1_{G=g^{i}} U\left(V_{T}^{1, H^{\mathbb{F}, i}}\right)\right]=\mathbb{E}^{\mathbb{P}}\left[U\left(V_{T}^{1, H^{\mathbb{G}}}\right)\right] \leq \sup _{H \in \mathscr{A}_{1}^{\mathbb{G}}} \mathbb{E}^{\mathbb{P}}\left[U\left(V_{T}^{1, H}\right)\right] .
$$

The following theorem leads to a new characterization of the expected utility of the insider in terms of the additional information $G$ and the set of all local martingale densities of the $(\mathbb{F}, \mathbb{P})$-market.

Theorem 3.8. Let $G$ be discrete, suppose that Assumptions 2.6 and 2.9 hold true, and assume that

(i) The function $U:(0, \infty) \rightarrow \mathbb{R}$ is strictly concave, increasing, continuously differentiable and satisfies the Inada conditions at 0 and $\infty$.

(ii) For every $y \in(0, \infty)$, there exists $Z \in E L M M D(\mathbb{F}, \mathbb{P})$ with $\mathbb{E}^{\mathbb{P}}[V(y Z)]<\infty$, where $V(y)=\sup _{x}(U(x)-x y)$.

Then,

$$
\sup _{H \in \mathscr{A} \mathbb{G}_{1}^{\mathbb{G}}} \mathbb{E}^{\mathbb{P}}\left[U\left(V_{T}^{1, H}\right)\right]=\sum_{i} \inf _{y>0}\left\{y+\inf _{Z \in E L M M D(\mathbb{F}, \mathbb{P})} \mathbb{E}^{\mathbb{P}}\left[V\left(y Z_{T}\right) 1_{G=g_{i}}\right]\right\} .
$$

Proof. In view of Lemma 3.7 it suffices to show that for every $i$,

$$
\sup _{H \in \mathscr{A}_{1}^{\mathbb{P}}} \mathbb{E}^{\mathbb{P}}\left[\mathbf{1}_{G=g_{i}} U\left(V_{T}^{1, H}\right)\right]=\inf _{y>0}\left\{y+\inf _{Z \in E L M M D(\mathbb{F}, \mathbb{P})} \mathbb{E}^{\mathbb{P}}\left[V\left(y Z_{T}\right) 1_{G=g_{i}}\right]\right\} .
$$

Following the reasoning in the latter part of the proof of Theorem 3.1 that relates the $\left(\mathbb{F}, \mathbb{Q}^{i}\right)$ - markets to the $(\mathbb{F}, \mathbb{P})$ - market and using Assumption 2.9, one can again use 
Theorem 4.1 in $[10]$ to show that the $\left(\mathbb{F}, \mathbb{Q}^{i}\right)$-market satisfies the condition NUPBR. Furthermore, for any local martingale density $Z \in E L M M D(\mathbb{F}, \mathbb{P})$, the process $Z / p^{g_{i}}$ is a local martingale deflator for the $\left(\mathbb{F}, \mathbb{Q}^{i}\right)$-market (note that on $\left\{G=g_{i}\right\}, p_{t}^{g_{i}}>0$ for all $t$ ).

Let us next introduce the following subsets of $L_{+}^{0}$

$$
\begin{aligned}
\mathscr{C}(x) & =\left\{v \in L_{+}^{0}: 0 \leq v \leq x V_{T}^{1, H^{\mathbb{F}}}, \mathbb{P}-\text { a.s. for some } H^{\mathbb{F}} \in \mathscr{A}_{1}^{\mathbb{F}}\right\}, \\
\mathscr{D}(y) & =\left\{z \in L_{+}^{0}: 0 \leq z \leq y Z_{T}, \mathbb{P}-\text { a.s. for some } Z \in E L M M(\mathbb{F}, \mathbb{P})\right\}, \\
\mathscr{C}^{i}(x) & =\left\{v^{i}=v, \mathbb{Q}^{i}-\text { a.s. for some } v \in \mathscr{C}(x)\right\}, \\
\mathscr{D}^{i}(y) & =\left\{z^{i}=\frac{z}{p_{T}^{g_{i}}}, \mathbb{Q}^{i}-\text { a.s. for some } z \in \mathscr{D}(y)\right\} .
\end{aligned}
$$

Because the $(\mathbb{F}, \mathbb{P})$-market was assumed to satisfy NFLVR, Proposition 3.1 of [28] implies that $\mathscr{C}$ and $\mathscr{D}$ are convex with the following properties

$$
\begin{aligned}
& v \in \mathscr{C}(1) \Longleftrightarrow \mathbb{E}^{\mathbb{P}}[v z] \leq 1, \text { for all } z \in \mathscr{D}(1), \\
& z \in \mathscr{D}(1) \Longleftrightarrow \mathbb{E}^{\mathbb{P}}[v z] \leq 1, \text { for all } v \in \mathscr{C}(1) .
\end{aligned}
$$

These imply that for every $i$,

$$
\begin{aligned}
v^{i} \in \mathscr{C}^{i}(1) & \Leftrightarrow \mathbb{E}^{\mathbb{Q}^{i}}\left[v^{i} z^{i}\right] \leq 1, \text { for all } z^{i} \in \mathscr{D}^{i}(1), \\
z^{i} \in \mathscr{D}^{i}(1) & \Leftrightarrow \mathbb{E}^{\mathbb{Q}^{i}}\left[v^{i} z^{i}\right] \leq 1, \text { for all } v^{i} \in \mathscr{C}^{i}(1) .
\end{aligned}
$$

and thus the assumption (3.1) of [31] holds for $\mathscr{C}^{i}(1)$ and $\mathscr{D}^{i}(1)$ under the measure $\mathbb{Q}^{i}$. In addition, $\mathscr{C}^{i}$ and $\mathscr{D}^{i}$ contain at least one strictly positive element.

Define, following [31], the optimization problems

$$
u(x)=\sup _{\xi \in \mathscr{C}^{i}(x)} \mathbb{E}^{\mathbb{Q}^{i}}[U(\xi)] \quad \text { and } \quad v(y)=\inf _{\eta \in \mathscr{D}^{i}(y)} \mathbb{E}^{\mathbb{Q}^{i}}[V(\eta)] .
$$

For all $y>0$, the finiteness of $v(y)$ follows from the assumptions of the Theorem. Furthermore, since $x \in \mathscr{C}^{i}(x)$, we have $u(x)>-\infty$ for all $x>0$. An application of Theorem 3.2 of [31] then shows that $u$ and $v$ satisfy biconjugacy relations so that in particular $u(x)=\inf _{y>0}(v(y)+x y)$. Taking $x=1$ and substituting the explicit expression for $\mathbb{Q}^{i}$, the proof is complete.

From Theorem 3.8, one immediately obtains more explicit expressions for the case of power and logarithmic utility functions.

Corollary 3.9. Fix $\gamma \in(0,1)$. Let $G$ be discrete, suppose that Assumptions 2.6 and 2.9 hold true, and that there exists $Z \in E L M M D(\mathbb{F}, \mathbb{P})$ with $\mathbb{E}^{\mathbb{P}}\left[\left(Z_{T}\right)^{-\frac{\gamma}{1-\gamma}}\right]<\infty$. Then,

$$
\sup _{H \in \mathscr{A}_{1}^{\mathbb{G}}} \mathbb{E}^{\mathbb{P}}\left[\left(V_{T}^{1, H}\right)^{\gamma}\right]=\sum_{i}\left\{\inf _{Z \in E L M M D(\mathbb{F}, \mathbb{P})} \mathbb{E}^{\mathbb{P}}\left[\left(Z_{T}\right)^{-\frac{\gamma}{1-\gamma}} 1_{G=g_{i}}\right]\right\}^{1-\gamma} .
$$

Corollary 3.10. Let $G$ be discrete, suppose that Assumptions 2.6 and 2.9 hold true, and that there exists $Z \in E L M M D(\mathbb{F}, \mathbb{P})$ with $\mathbb{E}^{\mathbb{P}}\left[\log Z_{T}\right]>-\infty$. Then

$$
\begin{aligned}
\sup _{H \in \mathscr{A}_{1}^{\mathbb{G}}} \mathbb{E}^{\mathbb{P}}\left[\log V_{T}^{1, H}\right]= & -\sum_{i} \mathbb{P}\left[G=g_{i}\right] \log \mathbb{P}\left[G=g_{i}\right] \\
& +\sum_{i} \inf _{Z \in E L M M D(\mathbb{F}, \mathbb{P})} \mathbb{E}^{\mathbb{P}}\left[1_{G=g_{i}} \log \frac{1}{Z_{T}}\right] .
\end{aligned}
$$


To conclude this subsection, we shall compare our result for the logarithmic utility with the results in [4]. Let the additional expected log-utility of the insider be denoted by

$$
\Delta(\mathbb{F}, \mathbb{G}):=\sup _{H \in \mathscr{A}_{1}^{\mathbb{G}}} \mathbb{E}^{\mathbb{P}}\left[\log V_{T}^{1, H}\right]-\sup _{H \in \mathscr{A}_{1}^{\mathbb{F}}} \mathbb{E}^{\mathbb{P}}\left[\log V_{T}^{1, H}\right] .
$$

In the approach of [4], the quantity $\Delta(\mathbb{F}, \mathbb{G})$ is represented by the information drift, see Definition 3.6 in their paper, and in our approach, it can be expressed as

$$
\begin{gathered}
-\sum_{i=1}^{n} \mathbb{P}\left[G=g_{i}\right] \log \mathbb{P}\left[G=g_{i}\right]+\sum_{i=1}^{n} \inf _{Z \in E L M M D(\mathbb{F}, \mathbb{P})} \mathbb{E}^{\mathbb{P}}\left[1_{G=g_{i}} \log \frac{1}{Z_{T}}\right] \\
-\inf _{Z \in E L M M D(\mathbb{F}, \mathbb{P})} \mathbb{E}^{\mathbb{P}}\left[\log \frac{1}{Z_{T}}\right] .
\end{gathered}
$$

If the market is complete, then the two approaches end up with the same result: the quantity in (6) reduces to the entropy of $G$, which is exactly what is stated in Theorem 4.1 of [4].

\subsection{Example}

In this subsection we present an explicit example which corresponds to an incomplete market. A complete market example based on the standard Brownian motion is given in [11].

Suppose that $N^{1}$ and $N^{2}$ are two independent Poisson processes with common intensity $\lambda=1$. We consider a financial market with the (discounted) risky asset price $S_{t}=e^{N_{t}^{1}-N_{t}^{2}}$ whose dynamics is given by

$$
d S_{t}=S_{t-}\left((e-1) d N_{t}^{1}+\left(e^{-1}-1\right) d N_{t}^{2}\right), \quad S_{0}=1, \quad t \in[0, T] .
$$

The public information $\mathbb{F}$ is generated by the two Poisson processes $N^{1}, N^{2}$. The $(\mathbb{F}, \mathbb{P})$ market satisfies the NFLVR condition, and the density $Z$ of any equivalent local martingale measure is of the form

$$
d Z_{t}=Z_{t-}\left(\left(\alpha_{t}^{1}-1\right)\left(d N_{t}^{1}-d t\right)+\left(\alpha_{t}^{2}-1\right)\left(d N_{t}^{2}-d t\right)\right),
$$

where $\alpha^{1}, \alpha^{2}$ are positive integrable processes satisfying $\alpha_{t}^{1}=e^{-1} \alpha_{t}^{2}$.

Let us define $N_{t}:=N_{t}^{1}-N_{t}^{2}$ and assume that the insider knows the value of $N_{T}$, and hence of $S_{T}$, at the beginning of trading. The insider's filtration is thus $\mathscr{G}_{t}=\mathscr{F}_{t} \mathrm{~V}$ $\sigma\left(N_{T}\right)=\mathscr{F}_{t} \vee \sigma\left(S_{T}\right)$. An easy computation shows that for all $t \in[0, T)$,

$$
p_{t}^{x}=\frac{\mathbb{P}\left[N_{T}=x \mid \mathscr{F}_{t}\right]}{\mathbb{P}\left[N_{T}=x\right]}=\frac{\sum_{k \geq 0} e^{-(T-t)} \frac{(T-t)^{k}}{k !} e^{-(T-t)} \frac{(T-t)^{k+x-N_{t}}}{\left(k+x-N_{t}\right) !} 1_{k+x-N_{t} \geq 0}}{\sum_{k \geq 0} e^{-T} \frac{T^{k}}{k !} e^{-T} \frac{T^{x+k}}{(x+k) !}}>0
$$

and for $t=T$

$$
p_{T}^{x}=\frac{1_{N_{T}=x}}{\sum_{k \geq 0} e^{-T} \frac{T^{k}}{k !} e^{-T} \frac{T^{x+k}}{(x+k) !}} .
$$

Since the density $p_{t}^{x}$ is strictly positive before time $T$, Assumption (2.9) is fulfilled. Theorem 3.1 allows then to conclude that the $(\mathbb{G}, \mathbb{P})$-market satisfies NUPBR. 


\subsubsection{Optimal arbitrage.}

By Theorem 3.3, the superhedging price of 1 under $\mathbb{G}$ is

$$
x_{*}^{\mathbb{G}, \mathbb{P}}(1)=\sum_{x \in \mathbb{Z}} x_{*}^{\mathbb{F}, \mathbb{P}}\left(1_{N_{T}=x}\right) 1_{N_{T}=x} .
$$

To check whether optimal arbitrage exists, we need to compute

$$
x_{*}^{\mathbb{F}, \mathbb{P}}\left(1_{N_{T}=x}\right)=\sup _{\overline{\mathbb{P}} \in \operatorname{ELMM}(\mathbb{F}, \mathbb{P})} \overline{\mathbb{P}}\left[N_{T}=x\right]
$$

for every $x \in \mathbb{Z}$. This is the goal of the following proposition.

Proposition 3.11. If $x \leq 0$, we have

$$
x_{*}^{\mathbb{F}, \mathbb{P}}\left(1_{N_{T}=x}\right)=\sup _{\overline{\mathbb{P}} \in \operatorname{ELMM}(\mathbb{F}, \mathbb{P})} \overline{\mathbb{P}}\left[N_{T}=x\right]=1
$$

and there is no optimal arbitrage. If $x>0$, we have that

$$
x_{*}^{\mathbb{F}, \mathbb{P}}\left(1_{N_{T}=x}\right)=\sup _{\mathbb{P} \in E L M M(\mathbb{F}, \mathbb{P})} \overline{\mathbb{P}}\left[N_{T}=x\right]=\frac{1}{e^{x}}
$$

and the optimal arbitrage strategy is the strategy in which the agent buys $\frac{1}{S_{T}}$ units of the risky asset and holds them until maturity.

Proof. First, we consider the case $x \leq 0$. Let us define $\tau=\inf \left\{t: N_{t}=x\right\}$. We fix two constants $M>m>0$ and choose $\alpha_{t}^{1}=M \mathbf{1}_{t \leq \tau}+m \mathbf{1}_{t>\tau}$. The process $\alpha_{t}^{1}$, and consequently also $\alpha_{t}^{2}$, is thus bounded implying that for the density $Z$ one has $\mathbb{E}^{\mathbb{P}}\left[Z_{T}\right]=$ 1 (see [9. Theorem VI.T4]). Denote by $\overline{\mathbb{P}}^{M, m}$ the corresponding martingale measure. For this measure, the following inequality holds true.

$$
\sup _{\overline{\mathbb{P}} \in \operatorname{ELMM}(\mathbb{F}, \mathbb{P})} \overline{\mathbb{P}}\left[N_{T}=x\right] \geq \mathbb{E}^{\overline{\mathbb{P}}^{M, m}}\left[1_{N_{T}=x}\right] \geq \overline{\mathbb{P}}^{M, m}\left[\tau \leq T ; N_{t}^{1}=N_{\tau}^{1}, N_{t}^{2}=N_{\tau}^{2} \forall t \in[\tau, T]\right] .
$$

By the strong Markov property, $N_{\tau+s}^{1}-N_{\tau}^{1}$ and $N_{\tau+s}^{2}-N_{\tau}^{2}$ are independent Poisson processes with intensities $m$ and $e m$, independent from $\mathscr{F} \tau$. Therefore,

$$
\begin{aligned}
\overline{\mathbb{P}}^{M, m}\left[\tau \leq T ; N_{t}^{1}=N_{\tau}^{1}, N_{t}^{2}=N_{\tau}^{2} \forall t \in[\tau, T]\right] & \geq \overline{\mathbb{P}}^{M, m}[\tau \leq T] \overline{\mathbb{P}}^{m}\left[N_{t}^{1}=0, N_{t}^{2}=0 \forall t \in[0, T]\right] \\
& =\overline{\mathbb{P}}^{M, m}[\tau \leq T] e^{-m(1+e) T},
\end{aligned}
$$

where $\overline{\mathbb{P}}^{m}$ is the probability measure under which $N^{1}$ is a Poisson process with intensity $m$ and $N^{2}$ is a Poisson process with intensity em. On the other hand, up to time $\tau, N^{1}$ and $N^{2}$ are independent Poisson processes with intensities $M$ and $e M$. Therefore,

$$
\overline{\mathbb{P}}^{M, m}[\tau \leq T]=\overline{\mathbb{P}}^{M}\left[\inf _{0 \leq t \leq T}\left(N_{t}^{1}-N_{t}^{2}\right) \leq x\right]=\overline{\mathbb{P}}^{1}\left[\inf _{0 \leq t \leq M T}\left(N_{t}^{1}-N_{t}^{2}\right) \leq x\right]
$$

Letting $m$ go to zero and $M$ go to infinity and using the dominated convergence theorem, we obtain

$$
\sup _{\overline{\mathbb{P}} \in \operatorname{ELMM}(\mathbb{F}, \mathbb{P})} \overline{\mathbb{P}}\left[N_{T}=x\right] \geq \overline{\mathbb{P}}^{1}\left[\inf _{t \geq 0}\left(N_{t}^{1}-N_{t}^{2}\right) \leq x\right]=1,
$$


because $N_{t}^{1}-N_{t}^{2} \rightarrow-\infty$ under $\overline{\mathbb{P}}^{1}$ as $t \rightarrow \infty$. So, the first statement holds true.

Coming next to the case $x>0$, we notice that $e^{-x}$ is an upper bound for the supremum. Indeed, for any ELMM $\mathbb{P}$, it holds that

$$
\overline{\mathbb{P}}\left[N_{T}=x\right] \leq \overline{\mathbb{P}}\left[S_{T} \geq e^{x}\right] \leq \frac{\mathbb{E}^{\overline{\mathbb{P}}}\left[S_{T}\right]}{e^{x}} \leq \frac{1}{e^{x}} .
$$

Repeating the computations as in the first case, we obtain

$$
\begin{aligned}
\sup _{\overline{\mathbb{P}} \in \operatorname{ELMM}(\mathbb{F}, \mathbb{P})} \overline{\mathbb{P}}\left[N_{T}=x\right] \geq \mathbb{E}^{\overline{\mathbb{P}}^{M, m}}\left[1_{N_{T}=x}\right] & =\overline{\mathbb{P}}^{M}[\tau \leq T] e^{-m(1+e) T} \\
& =\overline{\mathbb{P}}^{1}\left[\sup _{0 \leq t \leq M T}\left(N_{t}^{1}-N_{t}^{2}\right) \geq x\right] e^{-m(1+e) T} .
\end{aligned}
$$

It thus suffices to show that

$$
f(x):=\overline{\mathbb{P}}^{1}\left[\sup _{t \geq 0}\left(N_{t}^{1}-N_{t}^{2}\right) \geq x\right]=\frac{1}{e^{x}} .
$$

Let $\tau_{1}, \tau_{2}$ be the first jump times of $N^{1}$ and $N^{2}$, respectively. Because $\tau_{1} \sim \operatorname{Exp}(1)$ and $\tau_{2} \sim \operatorname{Exp}(e)$ are independent under $\overline{\mathbb{P}}^{1}$, the random variable $\frac{\tau_{1}}{e \tau_{2}}$ has the density $\frac{1}{(1+t)^{2}}$, thanks to Lemma4.12, and thus,

$$
\overline{\mathbb{P}}^{1}\left[\tau_{1}<\tau_{2}\right]=\overline{\mathbb{P}}^{1}\left[\frac{\tau_{1}}{e \tau_{2}}<\frac{1}{e}\right]=\int_{0}^{1 / e} \frac{1}{(1+t)^{2}} d t=\frac{1}{1+e} .
$$

From its definition, we have $f(0)=1$ and for $x \geq 1$ it then follows that

$$
\begin{aligned}
f(x) & =\overline{\mathbb{P}}^{1}\left[\sup _{t \geq 0}\left(N_{t}^{1}-N_{t}^{2}\right) \geq x \mid \tau_{1}>\tau_{2}\right] \mathbb{P}\left[\tau_{1}>\tau_{2}\right] \\
& +\overline{\mathbb{P}}^{1}\left[\sup _{t \geq 0}\left(N_{t}^{1}-N_{t}^{2}\right) \geq x \mid \tau_{1} \leq \tau_{2}\right] \mathbb{P}\left[\tau_{1} \leq \tau_{2}\right] \\
& =\frac{e f(x+1)}{1+e}+\frac{f(x-1)}{1+e} .
\end{aligned}
$$

Therefore, we obtain $f(x+1)-f(x)=\frac{f(x)-f(x-1)}{e}$ and thus

$$
f(x)=1-(1-f(1)) \frac{1-e^{-x}}{1-e^{-1}}
$$

Because $\lim _{x \rightarrow \infty} f(x)=0$, we have that $f(1)=e^{-1}$ and then $f(x)=e^{-x}$.

Now we show that the buy and hold strategy is optimal. Because the insider knows the value of $S_{T}=e^{N_{T}}$, the buy and hold strategy, consisting of $\frac{1}{S_{T}}$ units of the risky asset, superreplicates the claim 1 . In fact

$$
\frac{1}{S_{T}}+\frac{1}{S_{T}} \int_{0}^{T} 1 d S_{u}=1
$$

For this the insider needs the initial capital $e^{-x}$ on the event $N_{T}=x$. 


\subsubsection{Expected log-utility.}

By Corollary 3.10 the expected log-utility of the insider is

$\sup _{H \in \mathscr{A} \mathbb{G}^{\mathbb{G}}} \mathbb{E}^{\mathbb{P}}\left[\log V_{T}^{1, H}\right]=-\sum_{x \in \mathbb{Z}} \mathbb{P}\left[N_{T}=x\right] \log \mathbb{P}\left[N_{T}=x\right]-\sum_{x \in \mathbb{Z} Z \in E L M M D(\mathbb{F}, \mathbb{P})} \sup ^{\mathbb{P}}\left[1_{N_{T}=x} \log Z_{T}\right]$.

Choosing a specific strategy $H$ in the left-hand side, we obtain a lower bound for the $\log$ utility, and a specific equivalent martingale measure density $Z$ in the right-hand side provides an upper bound. We are going to construct $H$ and $Z$ for which the two bounds coincide. As a preliminary step, we are going to evaluate the intensities of $N^{1}$ and $N^{2}$ under $\mathbb{G}$.

Intensities of $N^{1}$ and $N^{2}$ under $\mathbb{G} \quad$ Let $\lambda^{\mathbb{G}, 1}, \lambda^{\mathbb{G}, 2}$ be the intensities of $N^{1}, N^{2}$ under $\mathbb{G}$, respectively. Introduce a further larger filtration $\mathscr{H}_{t}=\mathscr{F}_{t} \vee \sigma\left(N_{T}^{1}, N_{T}^{2}\right)$. Under $\mathbb{H}$, we obtain that

$$
d N_{t}^{1}-\frac{N_{T}^{1}-N_{t}^{1}}{T-t} d t, \quad d N_{t}^{2}-\frac{N_{T}^{2}-N_{t}^{2}}{T-t} d t
$$

are martingales, see Theorem VI.3 of [33]. Now, Lemma4.14] of the Appendix implies that the processes

$$
N_{t}^{1}-\int_{0}^{t} \mathbb{E}\left[\frac{N_{T}^{1}-N_{s}^{1}}{T-s} \mid \mathscr{G}_{S}\right] d s, \quad N_{t}^{2}-\int_{0}^{t} \mathbb{E}\left[\frac{N_{T}^{2}-N_{s}^{2}}{T-s} \mid \mathscr{G}_{S}\right] d s
$$

are martingales under $\mathbb{G}$ and so

$$
\begin{aligned}
& \lambda_{t}^{\mathbb{G}, 1}=\mathbb{E}\left[\frac{N_{T}^{1}-N_{t}^{1}}{T-t} \mid \mathscr{G}_{t}\right] \\
& =\frac{1}{T-t} \mathbb{E}\left[N_{T}^{1}-N_{t}^{1} \mid N_{T}^{1}-N_{t}^{1}-N_{T}^{2}+N_{t}^{2}\right]=\frac{1}{T-t} f_{t}^{1}\left(N_{T}^{1}-N_{t}^{1}-N_{T}^{2}+N_{t}^{2}\right),
\end{aligned}
$$

where

$$
f_{t}^{1}(y)=\mathbb{E}\left[N_{T-t}^{1} \mid N_{T-t}^{1}-N_{T-t}^{2}=y\right]=\frac{\mathbb{E}\left[N_{T-t}^{1} 1_{N_{T-t}^{1}-N_{T-t}^{2}=y}\right]}{\mathbb{P}\left[N_{T-t}^{1}-N_{T-t}^{2}=y\right]} .
$$

This computation can be made explicit. For example, if $y>0$,

$$
\begin{aligned}
f_{t}^{1}(y) & =\frac{\sum_{k \geq 0}(y+k) \mathbb{P}\left[N_{T-t}^{2}=k\right] \mathbb{P}\left[N_{T-t}^{1}=y+k\right]}{\sum_{k \geq 0} \mathbb{P}\left[N_{T-t}^{2}=k\right] \mathbb{P}\left[N_{T-t}^{1}=y+k\right]} \\
& =\frac{\sum_{k \geq 0}(y+k) \frac{(T-t)^{2 k+y}}{k !(y+k) !}}{\sum_{k \geq 0} \frac{(T-t)^{2 k+y}}{k !(k+y) !}}=\frac{(T-t) I_{y-1}(2(T-t))}{I_{y}(2(T-t))},
\end{aligned}
$$

where $I_{\alpha}(x)$ is the modified Bessel functions of the first kind1. A similar computation for $y \leq 0$ shows that for all integer $y$,

$$
f_{t}^{1}(y)=\frac{(T-t) I_{|y-1|}(2(T-t))}{I_{|y|}(2(T-t))},
$$

\footnotetext{
${ }^{1}$ The modified Bessel functions of the first kind is defined by the series representation $I_{\alpha}(x)=$ $\sum_{m \geq 0} \frac{1}{m ! \Gamma(m+\alpha+1)}\left(\frac{x}{2}\right)^{2 m+\alpha}$, for a real number $\alpha$ which is not a negative integer, and satisfies $I_{-n}(x)=I_{n}(x)$ for integer $n$
} 
so that finally

$$
\lambda_{t}^{\mathbb{G}, 1}=\frac{I_{\left|N_{T}-N_{t}-1\right|}(2(T-t))}{I_{\left|N_{T}-N_{t}\right|}(2(T-t))} \quad \text { and } \quad \lambda_{t}^{\mathbb{G}, 2}=\frac{I_{\left|N_{T}-N_{t}+1\right|}(2(T-t))}{I_{\left|N_{T}-N_{t}\right|}(2(T-t))} .
$$

Upper bound by duality From equation 17, we get

$$
\mathbb{E}^{\mathbb{P}}\left[1_{N_{T}=x} \log Z_{T}\right]=\mathbb{E}^{\mathbb{P}}\left[1_{N_{T}=x} \mathbb{E}^{\mathbb{P}}\left[\sum_{i=1}^{2} \int_{0}^{T} \log \alpha_{t}^{i} d N_{t}^{i}-\int_{0}^{T}\left(\alpha_{t}^{i}-1\right) d t \mid \mathscr{G}_{0}\right]\right] .
$$

Since we are only interested in an upper bound, we can restrict the discussion to equivalent local martingale densities for which

$$
\mathbb{E}\left[\int_{0}^{t} \log \alpha_{s}^{i}\left(d N_{s}^{i}-\lambda_{s}^{\mathbb{G}, i}\right) \mid \mathscr{G}_{0}\right]=0 \quad \text { a.s. }
$$

In this case, the above expectation becomes

$$
\begin{aligned}
\mathbb{E}^{\mathbb{P}}\left[1_{N_{T}=x} \log Z_{T}\right] & =\mathbb{E}^{\mathbb{P}}\left[1_{N_{T}=x} \sum_{i=1}^{2} \int_{0}^{T}\left(\lambda_{t}^{\mathbb{G}, i} \log \alpha_{t}^{i}-\left(\alpha_{t}^{i}-1\right)\right) d t\right] \\
& =\mathbb{E}^{\mathbb{P}}\left[1_{N_{T}=x} \int_{0}^{T}\left(\lambda_{t}^{\mathbb{G}, 1} \log \alpha_{t}^{1}-\alpha_{t}^{1}+\lambda_{t}^{\mathbb{G}, 2} \log \left(e \alpha_{t}^{1}\right)-e \alpha_{t}^{1}\right) d t\right]
\end{aligned}
$$

The expression

$$
\lambda_{t}^{\mathbb{G}, 1} \log \alpha_{t}^{1}-\alpha_{t}^{1}+\lambda_{t}^{\mathbb{G}, 2} \log \left(e \alpha_{t}^{1}\right)-e \alpha_{t}^{1}
$$

is concave as function of $\alpha_{t}^{1}$, with unique maximum attained at

$$
\frac{\lambda_{t}^{\mathbb{G}, 1}+\lambda_{t}^{\mathbb{G}, 2}}{e+1}
$$

Fix $\varepsilon>0$ and let $\alpha_{t}^{1}=\frac{\lambda_{t}^{\mathbb{G}, 1}+\lambda_{t}^{\mathbb{G}, 2}}{e+1} 1_{0 \leq t \leq T-\varepsilon}+1_{T-\varepsilon<t \leq T}$, and $\alpha_{t}^{2}=e \alpha_{t}^{1}$. These values are bounded on $[0, T]$ implying that the corresponding density satisfies $\mathbb{E}^{\mathbb{P}}\left[Z_{T}\right]=1$ and 11.). Plugging it into (12), we obtain

$$
\begin{aligned}
& \sup _{Z \in E L M M D(\mathbb{F}, \mathbb{P})} \mathbb{E}^{\mathbb{P}}\left[1_{N_{T}=x} \log Z_{T}\right] \geq \mathbb{E}^{\mathbb{P}}\left[1_{N_{T}=x} \int_{T-\varepsilon}^{T}\left(-1-e+\lambda_{t}^{\mathbb{G}, 2}\right) d t\right] \\
& +\mathbb{E}^{\mathbb{P}}\left[1_{N_{T}=x} \int_{0}^{T-\varepsilon}\left(\log \left(\frac{\lambda_{t}^{\mathbb{G}, 1}+\lambda_{t}^{\mathbb{G}, 2}}{e+1}\right)\left(\lambda_{t}^{\mathbb{G}, 1}+\lambda_{t}^{\mathbb{G}, 2}\right)-\lambda_{t}^{\mathbb{G}, 1}+2\right) d t\right] .
\end{aligned}
$$

When $\varepsilon \rightarrow 0$, the expression under the two expectations is bounded from below and converges monotonically to

$$
1_{N_{T}=x} \int_{0}^{T}\left(\log \left(\frac{\lambda_{t}^{\mathbb{G}, 1}+\lambda_{t}^{\mathbb{G}, 2}}{e+1}\right)\left(\lambda_{t}^{\mathbb{G}, 1}+\lambda_{t}^{\mathbb{G}, 2}\right)-\lambda_{t}^{\mathbb{G}, 1}+2\right) d t,
$$


which shows that

$$
\begin{aligned}
& \sum_{x \in \mathbb{Z} \in E \operatorname{ELMM}(\mathbb{F}, \mathbb{P})} \sup ^{\mathbb{P}}\left[1_{N_{T}=x} \log Z_{T}\right] \\
& \geq \mathbb{E}^{\mathbb{P}}\left[\int_{0}^{T}\left(\log \left(\frac{\lambda_{t}^{\mathbb{G}, 1}+\lambda_{t}^{\mathbb{G}, 2}}{e+1}\right)\left(\lambda_{t}^{\mathbb{G}, 1}+\lambda_{t}^{\mathbb{G}, 2}\right)-\lambda_{t}^{\mathbb{G}, 1}+2\right) d t\right],
\end{aligned}
$$

and in particular the right-hand side is finite (by the duality result).

\subsubsection{A lower bound by direct computation}

Let $\pi^{\mathbb{G}}$ be a $\mathbb{G}$-predictable strategy, which denotes the ratio invested in the risky asset, and let $V^{1, \pi^{\mathbb{G}}}$ be the corresponding self-financing wealth process that starts from $x=1$ and whose dynamics are

$$
\frac{d V_{t}^{1, \pi^{\mathbb{G}}}}{V_{t-}^{1, \pi^{\mathbb{G}}}}=\pi_{t}^{\mathbb{G}} \frac{d S_{t}}{S_{t-}}=\pi_{t}^{\mathbb{G}}\left((e-1) d N_{t}^{1}+\left(e^{-1}-1\right) d N_{t}^{2}\right)
$$

The logarithm of $V^{1, \pi^{\mathbb{G}}}$ satisfies

$$
\begin{aligned}
d \log V_{t}^{1, \pi^{\mathbb{G}}} & =\log \left(1+(e-1) \pi_{t}^{\mathbb{G}}\right) d N_{t}^{1}+\log \left(1+\left(e^{-1}-1\right) \pi_{t}^{\mathbb{G}}\right) d N_{t}^{2} \\
& =\left(\log \left(1+(e-1) \pi_{t}^{\mathbb{G}}\right) \lambda_{t}^{\mathbb{G}, 1}+\log \left(1+\left(e^{-1}-1\right) \pi_{t}^{\mathbb{G}}\right) \lambda_{t}^{\mathbb{G}, 2}\right) d t+\mathbb{G} \text {-local martingale. }
\end{aligned}
$$

Since we are only looking for a lower bound, we can restrict the discussion to strategies for which the coefficients in front of $N^{1}$ and $N^{2}$ are bounded and the local martingale above is a true martingale. Taking the expectation of both sides, we obtain that

$$
\mathbb{E}^{\mathbb{P}}\left[\log V_{T}^{1, \pi^{\mathbb{G}}}\right]=\mathbb{E}^{\mathbb{P}}\left[\int_{0}^{T}\left(\log \left(1+(e-1) \pi_{t}^{\mathbb{G}}\right) \lambda_{t}^{\mathbb{G}, 1}+\log \left(1+\left(e^{-1}-1\right) \pi_{t}^{\mathbb{G}}\right) \lambda_{t}^{\mathbb{G}, 2}\right) d t\right] .
$$

The expression under the integral sign is concave in $\pi_{t}^{\mathbb{G}}$ and is maximized by

$$
\frac{\lambda_{t}^{\mathbb{G}, 1}(e-1)+\lambda_{t}^{\mathbb{G}, 2}\left(e^{-1}-1\right)}{(e-1)\left(1-e^{-1}\right)\left(\lambda_{t}^{\mathbb{G}, 1}+\lambda_{t}^{\mathbb{G}, 2}\right)} \in\left(-\frac{1}{e-1}, \frac{1}{1-e^{-1}}\right) .
$$

Fix $\varepsilon \in(0,1)$, define $\tau_{\varepsilon}=\inf \left\{t>0: \lambda_{t}^{\mathbb{G}, 1} \notin\left(\varepsilon, \varepsilon^{-1}\right)\right.$ or $\left.\lambda_{t}^{\mathbb{G}, 2} \notin\left(\varepsilon, \varepsilon^{-1}\right)\right\} \wedge T$ and let

$$
\pi_{t}^{\mathbb{G}}=\frac{\lambda_{t}^{\mathbb{G}, 1}(e-1)+\lambda_{t}^{\mathbb{G}, 2}\left(e^{-1}-1\right)}{(e-1)\left(1-e^{-1}\right)\left(\lambda_{t}^{\mathbb{G}, 1}+\lambda_{t}^{\mathbb{G}, 2}\right)} 1_{t \leq \tau_{\varepsilon}}
$$


Substituting this into (14), we then get

$$
\begin{aligned}
\mathbb{E}^{\mathbb{P}}\left[\log V_{T}^{1, \pi^{\mathbb{G}}}\right] & =\mathbb{E}^{\mathbb{P}}\left[\int_{0}^{\tau_{\varepsilon}}\left(\lambda_{t}^{\mathbb{G}, 1} \log \frac{(e+1) \lambda_{t}^{\mathbb{G}, 1}}{\lambda_{t}^{\mathbb{G}, 1}+\lambda_{t}^{\mathbb{G}, 2}}+\lambda_{t}^{\mathbb{G}, 2} \log \frac{\left(1+e^{-1}\right) \lambda_{t}^{\mathbb{G}, 2}}{\lambda_{t}^{\mathbb{G}, 1}+\lambda_{t}^{\mathbb{G}, 2}}\right) d t\right] \\
& \left.=-\mathbb{E}^{\mathbb{P}}\left[\int_{0}^{\tau_{\varepsilon}}\left(\lambda_{t}^{\mathbb{G}, 1}+\lambda_{t}^{\mathbb{G}, 1}\right) \log \frac{\lambda_{t}^{\mathbb{G}, 1}+\lambda_{t}^{\mathbb{G}, 2}}{e+1}-\lambda_{t}^{\mathbb{G}, 1}+2\right) d t\right] \\
& +\mathbb{E}^{\mathbb{P}}\left[\int_{0}^{\tau_{\varepsilon}}\left(\lambda_{t}^{\mathbb{G}, 1} \log \lambda_{t}^{\mathbb{G}, 1}+\lambda_{t}^{\mathbb{G}, 2} \log \left(\lambda_{t}^{\mathbb{G}, 1}\right)-\lambda_{t}^{\mathbb{G}, 1}-\lambda_{t}^{\mathbb{G}, 2}+2\right) d t\right] .
\end{aligned}
$$

When $\varepsilon \rightarrow 0, \tau_{\varepsilon} \rightarrow T$ and the first expectation above clearly converges to 13). To finish the proof, it remains to check that

$\mathbb{E}^{\mathbb{P}}\left[\int_{0}^{T}\left(\lambda_{t}^{\mathbb{G}, 1} \log \lambda_{t}^{\mathbb{G}, 1}+\lambda_{t}^{\mathbb{G}, 2} \log \left(\lambda_{t}^{\mathbb{G}, 1}\right)-\lambda_{t}^{\mathbb{G}, 1}-\lambda_{t}^{\mathbb{G}, 2}+2\right) d t \mid N_{T}=x\right]=-\log \mathbb{P}\left[N_{T}=x\right]$,

for $x \in \mathbb{Z}$.

To this end, let $M_{t}=\mathbb{P}\left[N_{T}=x \mid \mathscr{F}_{t}\right]$. By direct computation, we obtain that $M_{t}=$ $e^{-2(T-t)} I_{\left|x-N_{t}\right|}(2(T-t))$. The change of variable formula then yields

$M_{T}=M_{0}+\int_{0}^{T} e^{-2(T-t)}\left(I_{\left|x-N_{t}-1\right|}-I_{\left|x-N_{t}\right|}\right)\left(d N_{t}^{1}-d t\right)+\int_{0}^{T} e^{-2(T-t)}\left(I_{\left|x-N_{t}+1\right|}-I_{\left|x-N_{t}\right|}\right)\left(d N_{t}^{2}-d t\right)$,

and further, on the event $N_{T}=x$,

$$
\log M_{T}=\log M_{0}+\int_{0}^{T} \log \lambda_{t}^{\mathbb{G}, 1} d N_{t}^{1}+\int_{0}^{T} \log \lambda_{t}^{\mathbb{G}, 2} d N_{t}^{2}-\int_{0}^{T}\left(\lambda_{t}^{\mathbb{G}, 1}+\lambda_{t}^{\mathbb{G}, 2}-2\right) d t .
$$

Finally, we may conclude that

$$
\begin{aligned}
& -\log \mathbb{P}\left[N_{T}=x\right]=-\log M_{0}=-\mathbb{E}\left[\log M_{0} \mid N_{T}=x\right] \\
& =\mathbb{E}\left[\int_{0}^{T} \log \lambda_{t}^{\mathbb{G}, 1} d N_{t}^{1}+\int_{0}^{T} \log \lambda_{t}^{\mathbb{G}, 2} d N_{t}^{2}-\int_{0}^{T}\left(\lambda_{t}^{\mathbb{G}, 1}+\lambda_{t}^{\mathbb{G}, 2}-2\right) d t \mid N_{T}=x\right] \\
& =\mathbb{E}\left[\int_{0}^{T} \log \lambda_{t}^{\mathbb{G}, 1} \lambda_{t}^{\mathbb{G}, 1} d t+\int_{0}^{T} \log \lambda_{t}^{\mathbb{G}, 2} \lambda_{t}^{\mathbb{G}, 2} d t-\int_{0}^{T}\left(\lambda_{t}^{\mathbb{G}, 1}+\lambda_{t}^{\mathbb{G}, 2}-2\right) d t \mid N_{T}=x\right]
\end{aligned}
$$

provided we may show that

$$
\mathbb{E}\left[\int_{0}^{T} \log ^{2} \lambda_{t}^{\mathbb{G}, 1} \lambda_{t}^{\mathbb{G}, 1} d t\right]<\infty .
$$

To see this, observe that from the asymptotics of the Bessel function it follows that

$$
\left|\log \lambda_{t}^{\mathbb{G}, 1}\right| \leq C|\log (T-t)| \log \left(1+\left|N_{T}-N_{t}\right|\right) .
$$

Plugging this into the above estimate and using (10), we finally have

$$
\mathbb{E}\left[\int_{0}^{T} \log ^{2} \lambda_{t}^{\mathbb{G}, 1} \lambda_{t}^{\mathbb{G}, 1} d t\right] \leq \mathbb{E}\left[\int_{0}^{T} C^{2}\left|\log ^{2}(T-t)\right| \log ^{2}\left(1+\left|N_{T}-N_{t}\right|\right) \frac{N_{T}^{1}-N_{t}^{1}}{T-t} d t\right]
$$

which is easily shown to be bounded (use Cauchy-Schwarz inequality plus the estimate $\mathbb{E}\left[N_{T}^{\alpha}\right]=O(T)$ for $\left.\alpha>0\right)$. 


\section{Initial enlargement with a general random variable}

We now consider the case when the $\mathscr{F}_{T}$-measurable random variable $G$, which represents the insider information, is not purely atomic as it was the case in the previous section.

It is usually observed that in this case the value of logarithmic utility of the insider is infinite, as for example in Theorem 4.4 of [32] where the insider has exact information about at least one stock's terminal price, or in [4] where the insider's additional expected logarithmic utility is related to the entropy of $G$. This difficulty appears at $T$, the time when the conditional law of $G$ given $\mathscr{F}_{T}$ is a Dirac measure and hence Jacod's condition fails.

Since, as we saw before, NUPBR is the minimal condition for well-posed expected utility maximization problems, it is useful to have conditions for NUPBR to hold. [1] and [2] give a sufficient condition so that NUPBR holds under $\mathbb{G}$ in infinite time horizon settings. Their idea is that, if the processes $p^{x}$ and $S$ do not jump to zero at the same time, then one can construct an equivalent (local) martingale deflator (ELMD) under $\mathbb{G}$ and it is known that the existence of such an ELMD implies NUPBR. However, in finite horizon settings, it may happen that the process $p^{x}$ is not well-defined at $T$, making it impossible to define an ELMD because $p^{x}$ appears in the denominator of such an ELMD.

In this paper we consider a finite horizon $T>0$ and, in the present section, we shall study arbitrage properties and expected utility maximization for the case of a non-atomic $G$. Before coming to utility maximization, in the next subsection we show that if $G$ is non-atomic and the set of local martingale densities $\operatorname{ELMMD}(\mathbb{F}, \mathbb{P})$ is uniformly integrable, then there always exists an arbitrage of the first kind and so NUPBR fails. It shows in particular that, if the $(\mathbb{F}, \mathbb{P})$ market is complete, then NUPBR always fails under $\mathbb{G}$. This negative message implies that the non-uniform integrability of $E L M M D(\mathbb{F}, \mathbb{P})$ is a necessary condition for NUPBR under $\mathbb{G}$. This result is then accompanied by two examples: one where the set of local martingale densities $\operatorname{ELMMD}(\mathbb{F}, \mathbb{P})$ is uniformly integrable, and one where it is not. In the second example the expected log-utility is finite, which gives (see Proposition 3.6) a sufficient condition for NUPBR to hold. On the other hand, in this second example the insider has non-scalable arbitrage opportunities so that NFLVR cannot hold. Initial filtration enlargement with a non-atomic $\mathscr{F}_{T}$-measurable random variable may therefore lead to viable market models which allow for economically meaningful unscalable arbitrages.

\subsection{Arbitrage of the first kind}

The following result does not require Assumption 2.9

Proposition 4.1. (Arbitrage of the first kind) Assume that

- The law of $G$ is not purely atomic,

- The set of densities of equivalent local martingale measures $\operatorname{ELMMD}(\mathbb{F}, \mathbb{P})$ is uniformly integrable.

Then there exists an arbitrage of the first kind for the insider.

Proof. Let us choose $B \subset \mathbb{R}$ such that $B$ does not contain any atoms of $G$ and $\mathbb{P}[G \in$ $B]=c>0$. For each $n$, let $\left(B_{i}^{n}\right)_{1 \leq i \leq n}$ be a partition of $B$ such that $\mathbb{P}\left[G \in B_{i}^{n}\right]=c / n$. We are going to show that $1_{G \in B}$ is an arbitrage of the first kind, in the sense of Definition 
2.4 First, consider the superhedging price of $1_{G \in B_{i}^{n}}$ and its associated hedging strategy $H^{\mathbb{F}, i}$ in the $(\mathbb{F}, \mathbb{P})$-market (see Corollary 10 in [13]), for which

$$
\sup _{Z \in E L M M D(\mathbb{F}, \mathbb{P})} \mathbb{E}^{\mathbb{P}}\left[Z_{T} 1_{G \in B_{i}^{n}}\right]+\left(H^{\mathbb{F}, i} \cdot S\right)_{T} \geq 1_{G \in B_{i}^{n}}
$$

Therefore,

$$
\sum_{i=1}^{n} \sup _{Z \in E L M M D(\mathbb{F}, \mathbb{P})} \mathbb{E}^{\mathbb{P}}\left[Z_{T} 1_{G \in B_{i}^{n}}\right] 1_{G \in B_{i}^{n}}+\left(\left(\sum_{i=1}^{n} H^{\mathbb{F}, i} 1_{G \in B_{i}^{n}}\right) \cdot S\right)_{T} \geq 1_{G \in B} .
$$

Because the set of all local martingale densities $\left\{Z_{T}: Z \in E L M M D(\mathbb{F}, \mathbb{P})\right\}$ is uniformly integrable, for any $\varepsilon>0$ there exists $K>0$ such that

$$
\sup _{Z \in E L M M D(\mathbb{F}, \mathbb{P})} \mathbb{E}^{\mathbb{P}}\left[Z_{T} 1_{Z_{T}>K}\right] \leq \varepsilon .
$$

The initial capital in (15) can then be estimated by

$$
\begin{aligned}
& \sum_{i=1}^{n} \sup _{Z \in E L M M D(\mathbb{F}, \mathbb{P})}\left(\mathbb{E}^{\mathbb{P}}\left[Z_{T} 1_{Z>K} 1_{G \in B_{i}^{n}}\right]+\mathbb{E}^{\mathbb{P}}\left[Z_{T} 1_{Z_{T} \leq K} 1_{G \in B_{i}^{n}}\right]\right) 1_{G \in B_{i}^{n}} \\
& \leq \sum_{i=1}^{n}\left(\varepsilon+K \mathbb{P}\left[G \in B_{i}^{n}\right]\right) 1_{G \in B_{i}^{n}}=\sum_{i=1}^{n}\left(\varepsilon+K \frac{c}{n}\right) 1_{G \in B_{i}^{n}} .
\end{aligned}
$$

We can choose $\varepsilon$ and $n$ such that the initial capital in (15) is arbitrarily small and thus the random variable $1_{G \in B}$ is an arbitrage of the first kind.

Remark 4.2 (A comparison with [4]). The above proposition extends Theorem 4.4 of [4]. More precisely, [4] show that the insider's additional expected logarithmic utility up to time T becomes infinite (which implies that NUPBR fails). However, their results apply only to continuous processes and require an even stronger condition than market completeness, namely that the inverse of $p^{G}$ may be represented as a stochastic integral, see condition (45) therein. By our result, we are able to construct unbounded profits in general market settings. In particular, the following example shows that the property of uniform integrability may also hold for some incomplete market models.

Example 4.3 (Incomplete market with uniformly integrable set of equivalent martingale densities). We consider a risky asset whose (discounted) price evolves as

$$
d S_{t}=S_{t-} \sigma(t)\left(\theta d N_{t}^{1}+(1-\theta) d N_{t}^{2}-d t\right)
$$

where $\theta \in(0,1)$ and $\sigma(t)$ is a continuous function which is not constant on $[0, T]$. The filtration $\mathbb{F}$ is generated by the two independent standard Poisson processes $N^{1}$ and $N^{2}$. Any martingale density has the form (7) where now $\alpha^{1}$ and $\alpha^{2}$ are positive predictable integrable processes satisfying $\theta \alpha_{t}^{1}+(1-\theta) \alpha_{t}^{2}=1, \mathbb{P}-$ a.s. Therefore,

$$
0 \leq \alpha^{1} \leq \frac{1}{\theta}, \quad 0 \leq \alpha^{2} \leq \frac{1}{1-\theta} .
$$

These inequalities lead to an upper bound for all martingale densities:

$$
Z_{T}=e^{-\int_{0}^{T}\left(\alpha_{t}^{1}+\alpha_{t}^{2}-2\right) d t} \prod_{i=1}^{N_{T}^{1}} \alpha_{t_{i}}^{1} \prod_{j=1}^{N_{T}^{2}} \alpha_{t_{j}}^{2} \leq e^{2 T} \frac{1}{\theta^{N_{T}^{1}}} \frac{1}{(1-\theta)^{N_{T}^{2}}} .
$$


As a result, the set of martingale densities is uniformly integrable. Let $\left(T_{i}^{1}\right)_{i>1}$ and $\left(T_{j}^{2}\right)_{j \geq 1}$ be the jump times of $N^{1}$ and $N^{2}$ respectively. Because $\sigma$ is a continuous function, the random variables $\sigma\left(T_{i}^{1}\right), \sigma\left(T_{j}^{2}\right)$ are continuous and not constant. This means that the random variable

$$
G=S_{T}=\exp \left(-\int_{0}^{T} \sigma(s) d s\right) \prod_{i=1}^{N_{T}^{1}}\left(1+\theta \sigma\left(T_{i}^{1}\right)\right) \prod_{j=1}^{N_{T}^{2}}\left(1+(1-\theta) \sigma\left(T_{j}^{2}\right)\right)
$$

is a non-atomic random variable for which the market of the insider does not satisfy $N U P B R$.

In the literature there are examples of incomplete market models where NUPBR holds and therefore the set of equivalent martingale densities is not uniformly integrable. The following one is due to [27].

Example 4.4 (Incomplete market where NUPBR holds but NFLVR fails). The market model in this example consists of a risk-free asset paying zero interest and a single risky asset driven by a Lévy process with two-sided jumps. The public information $\mathbb{F}$ is the natural filtration generated by a Brownian motion $W$ and two independent Poisson processes $N^{1}, N^{2}$ with common intensity $\lambda=1$. The risky asset is $S_{t}=\exp \left(M_{t}\right)$ where $M_{t}=W_{t}+N_{t}^{1}-N_{t}^{2}$ is a $\mathbb{F}$-martingale. The dynamics of $S$ under $\mathbb{F}$ is

$$
d S_{t}=S_{t-}\left(d W_{t}+\frac{1}{2} d t+(e-1) d N_{t}^{1}+\left(e^{-1}-1\right) d N_{t}^{2}\right) .
$$

Let $H^{\mathbb{F}}$ be an $\mathbb{F}$ - self-financing strategy and denote by $\pi^{\mathbb{F}}$ the fraction of wealth invested in the stock. The associated wealth is $V^{v, \pi^{\mathbb{F}}}$ satisfying

$$
\frac{d V_{t}^{v, \pi^{\mathbb{F}}}}{V_{t-}^{v, \pi^{\mathbb{F}}}}=\pi_{t}^{\mathbb{F}} \frac{d S_{t}}{S_{t-}}, \quad V_{0}^{v, \pi^{\mathbb{F}}}=v .
$$

The strategy $\pi^{\mathbb{F}}$ is admissible if for all $t \in[0, T]$ we have that $V_{t}^{v, \pi^{\mathbb{F}}} \geq 0, \mathbb{P}-$ a.s. The insider has the additional information given by the final value of $S$ so that $\mathscr{G}_{t}=\mathscr{F}_{t} \mathrm{~V}$ $\sigma\left(S_{T}\right)=\mathscr{F}_{t} \vee \sigma\left(M_{T}\right)$. In [27] it is shown that all admissible strategies are bounded and the expected logarithmic utility for an insider is bounded from above. This entails that the $(\mathbb{G}, \mathbb{P})$-market satisfies NUPBR (see Proposition 3.6). Furthermore, the insider has arbitrage opportunities since he knows the final value of $S$. For example, if he knows that $S_{T}>1$, which happens with positive probability, he could buy the asset $S$ and hold it until maturity; being $S_{0}=1$, this implies a riskless profit.

\subsection{An approximation procedure}

Assume that we are given a non purely atomic random variable $G \in \mathbb{R}$ representing the information of the insider. Let $\left\{\Gamma_{i}^{n}, i=1, \ldots, n\right\}$ be a finite increasing partition of $\mathbb{R}^{+}$ and denote

$$
\sigma\left(G^{n}\right)=\sigma\left(\left\{G \in \Gamma_{i}^{n}\right\}, i=1, \ldots, n\right) .
$$

We approximate $\sigma(G)$ by the increasing sequence of sigma algebras $\sigma\left(G^{n}\right)$

$$
\sigma\left(G^{n}\right) \subset \sigma\left(G^{n+1}\right) \subset \ldots \sigma(G)=\sigma\left(\bigcup_{n \geq 1} \sigma\left(G^{n}\right)\right)
$$


and define an increasing sequence of filtrations $\mathbb{G}^{n}=\left(\mathscr{G}_{t}^{n}\right)_{t \in[0 . T]}$, where $\mathscr{G}_{t}^{n}=\bigcap_{\varepsilon>0} \mathscr{F}_{t+\varepsilon} \vee$ $\sigma\left(G^{n}\right)$. For each $n$, we shall use the results from Section 3 to compute the expected utility under $\mathbb{G}^{n}$, the information at level $n$. The convergence properties given below in this section will then enable us to obtain the corresponding results under $\mathbb{G}$.

First we have to make sure that for each $\mathbb{G}^{n}$-market we can indeed apply the results from Section 3 which are shown under Assumption 2.9. Since in the $\mathbb{G}^{n}$-market the insider's information is given by $\sigma\left(\left\{G \in \Gamma_{i}^{n}\right\}, i=1, \cdots, n\right)$, we need to show that for all integers $n$ and $i$ the quantities $\frac{\mathbb{P}\left[G \in \Gamma_{i}^{n} \mid \mathscr{F}_{t}\right]}{\mathbb{P}\left[G \in \Gamma_{i}^{n}\right]}$ do not jump to zero. This is the object of the following assumption.

Assumption 4.5. For every $a<b$, the $\mathbb{F}$-martingale

$$
p_{t}^{(a, b)}(\omega):=\frac{\mathbb{P}\left[G \in(a, b) \mid \mathscr{F}_{t}\right](\omega)}{\mathbb{P}[G \in(a, b)]}=\frac{1}{\mathbb{P}[G \in(a, b)]} \int_{a}^{b} p_{t}^{x}(\omega) \mathbb{P}[G \in d x]
$$

does not jump to zero.

The main result of this subsection is now the following convergence result

Proposition 4.6. For any $\mathbb{G}$-predictable strategy $H^{\mathbb{G}}$, there exists a sequence of $\left(\mathbb{G}^{n}\right)$ predictable strategies $\left(H^{\mathbb{G}^{n}}\right)_{n}$ such that for every $t \in[0, T], H_{t}^{\mathbb{G}^{n}} \rightarrow H_{t}^{\mathbb{G}}$ almost surely.

Proof. By definition of the predictable $\sigma$-field, we can find a sequence of càglàd $\mathbb{G}$ adapted processes $\left(H^{\mathbb{G}, n}\right)_{n}$ such that for every $t \in[0, T], H_{t}^{\mathbb{G}, n} \rightarrow H_{t}^{\mathbb{G}}$ almost surely. Moreover, without loss of generality, the processes $\left(H^{\mathbb{G}, n}\right)_{n}$ may be assumed to be bounded.

Let $\left\{T_{i}^{n}\right\}_{0 \leq i \leq n}^{n \geq 1}$ be a sequence of (deterministic) partitions of the interval $[0, T]$ such that for every $n$,

$$
0=T_{0}^{n}<T_{1}^{n}<\cdots<T_{n}^{n}=T
$$

and $\lim _{n \rightarrow \infty} \max _{0 \leq i \leq n-1}\left|T_{i+1}^{n}-T_{i}^{n}\right|=0$. For a càglàd $\mathbb{G}$-adapted process $X$, define the process $X^{n}$ by $X_{0}^{n}=X_{0}$ and

$$
X_{t}^{n}=\sum_{i=0}^{n-1} X_{T_{i}} 1_{\left[T_{i}, T_{i+1}\right]}(t),
$$

for $0<t \leq T$. Then, $X^{n}$ is $\mathbb{G}$-adapted and for every $t \in[0, T], X_{t}^{n} \rightarrow X_{t}$ almost surely.

Finally, let $Y$ be a process of the form

$$
Y_{t}=\sum_{i=0}^{k} y_{T_{i}} 1_{\left[T_{i}, T_{i+1}\right]}(t)
$$

where, for each $i, y_{T_{i}}$ is a bounded $\mathscr{G}_{T_{i}}$ measurable random variable, and $T_{i}$ is a deterministic time such that $0=T_{0}<T_{1}<\cdots<T_{n}=T$. We define

$$
Y_{t}^{\mathbb{G}^{n}}:=\sum_{i=1}^{k} \mathbb{E}\left[y_{T_{i}} \mid \mathscr{G}_{T_{i}}^{n}\right] 1_{] T_{i}, T_{i+1}\right]}(t) .
$$

The process $Y^{\mathbb{G}^{n}}$ is $\mathbb{G}^{n}$-predictable. Using Lévy's "Upward" Theorem (see Theorem II.50.3 of [34]), we obtain $\mathbb{E}\left[y_{T_{i}} \mid \mathscr{G}_{T_{i}}^{n}\right] \rightarrow y_{T_{i}}, \mathbb{P}$ - a.s., which means that for every $t \in$ $[0, T], Y_{t}^{\mathbb{G}^{n}} \rightarrow Y_{t}$ almost surely. Combining the three approximations described above gives the proof of the proposition. 


\subsection{Expected utility maximization}

Analogously to subsection 3.2 we consider now the expected utility maximization and a dual representation of the optimal value for the case of a non purely atomic $G$. Based on Proposition 3.6, this will then allow one to show NUPBR under Assumption 2.9 by using the finiteness of expected utility.

We start with the main convergence result

Theorem 4.7. Let $U: \mathbb{R} \rightarrow \mathbb{R}_{+}$be increasing and continuous and suppose that $S$ is a continuous semimartingale. Then,

$$
\lim _{n \rightarrow \infty} \sup _{H \in \mathscr{A}_{1} \mathbb{G}^{n}} \mathbb{E}^{\mathbb{P}}\left[U\left(V_{T}^{1, H}\right)\right]=\sup _{H \in \mathscr{A}_{1}^{\mathbb{G}}} \mathbb{E}^{\mathbb{P}}\left[U\left(V_{T}^{1, H}\right)\right]
$$

Proof. The inequality $\leq$ is trivial since $U$ is increasing and $\mathscr{A}_{1}^{\mathbb{G}^{n}} \subset \mathscr{A}_{1}^{\mathbb{G}}$. To prove the opposite inequality, we choose $H^{\mathbb{G}} \in \mathscr{A}_{1}^{\mathbb{G}}$. Our first aim is to show that $H^{\mathbb{G}}$ may be supposed to be bounded. Indeed, the stochastic integral $H^{\mathbb{G}} \cdot S$ is defined as the $\mathscr{H}^{2}$ limit of $H \mathbf{1}_{|H| \leq n} \cdot S=H^{+} \mathbf{1}_{|H| \leq n} \cdot S-H^{-} \mathbf{1}_{|H| \leq n} \cdot S$, see [33, Section IV.2], and by the dominated convergence theorem for stochastic integrals [33, Theorem IV.32], this limit is attained uniformly in compacts in probability, and hence also almost surely along a subsequence. Introduce the stopping time

$$
\tau_{\varepsilon}^{n}=\inf \left\{t>0: 1+\varepsilon+\int_{0}^{t} H \mathbf{1}_{|H| \leq n} d S \leq 0\right\} \wedge T .
$$

Observe that

$$
1+\varepsilon+\int_{0}^{t \wedge \tau_{\varepsilon}^{n}} H \mathbf{1}_{|H| \leq n} d S \geq 0, \quad t \in[0, T]
$$

which means that the strategy defined by $\frac{1}{1+\varepsilon} H \mathbf{1}_{|H| \leq n} \mathbf{1}_{t \leq \tau_{\varepsilon}^{n}}$ belongs to $\mathscr{A}_{1}^{\mathbb{G}}$. The uniform convergence of the stochastic integrals implies that $\mathbb{P}\left[\tau_{\varepsilon}^{n}=T\right] \rightarrow 1$ as $n \rightarrow \infty$, and also

$$
1+\int_{0}^{T} \frac{1}{1+\varepsilon} H \mathbf{1}_{|H| \leq n} \mathbf{1}_{t \leq \tau_{\varepsilon}^{n}} d S \rightarrow 1+\frac{1}{1+\varepsilon} \int_{0}^{T} H_{t} d S_{t}
$$

as $n \rightarrow \infty$. By Fatou's lemma we then have

$$
\mathbb{E}\left[U\left(1+\frac{1}{1+\varepsilon} \int_{0}^{T} H_{t} d S_{t}\right)\right] \leq \liminf _{n} \mathbb{E}\left[U\left(1+\int_{0}^{T} \frac{1}{1+\varepsilon} H \mathbf{1}_{|H| \leq n} \mathbf{1}_{t \leq \tau_{\varepsilon}^{n}} d S\right)\right],
$$

and another application of Fatou's lemma shows that

$$
\mathbb{E}\left[U\left(1+\int_{0}^{T} H_{t} d S_{t}\right)\right] \leq \liminf _{\varepsilon \downarrow 0} \mathbb{E}\left[U\left(1+\frac{1}{1+\varepsilon} \int_{0}^{T} H_{t} d S_{t}\right)\right] .
$$

This argument shows that

$$
\sup _{H \in \mathscr{A}_{1}^{\mathbb{G}}} \mathbb{E}^{\mathbb{P}}\left[U\left(V_{T}^{1, H}\right)\right]=\sup _{H \in \overline{\mathscr{A}}_{1}^{\mathbb{G}}} \mathbb{E}^{\mathbb{P}}\left[U\left(V_{T}^{1, H}\right)\right]
$$

where $\overline{\mathscr{A}}_{1}^{\mathbb{G}}$ denotes the strategies in $\mathscr{A}_{1}^{\mathbb{G}}$ which are bounded by a deterministic constant. Therefore, from now on we may (and will) assume $H$ to be bounded by a constant $C$.

Now let $\left(H_{n}\right)$ be a sequence of strategies approximating $H$ in the sense of Proposition 4.6, which may be assumed to be bounded by the same constant $C$. Once again, by the dominated convergence theorem for stochastic integrals, we show that $H_{n} \cdot S$ 
converges to $H \cdot S$ uniformly on compacts in probability, and hence also almost surely along a subsequence. Similarly to the previous part, we construct an admissible strategy from $H_{n}$ by stopping it at a suitable stopping time. An application of Fatou's lemma then shows that

$$
\liminf _{n \rightarrow \infty} \sup _{H \in \mathscr{A}_{1}^{\mathbb{G}^{n}}} \mathbb{E}^{\mathbb{P}}\left[U\left(V_{T}^{1, H}\right)\right] \geq \sup _{H \in \mathscr{A}_{1}^{\mathbb{G}}} \mathbb{E}^{\mathbb{P}}\left[U\left(V_{T}^{1, H}\right)\right] .
$$

Observing that the expression under the limit in the left hand side is increasing in $n$ and combining this with the opposite inequality, we conclude the proof.

Remark 4.8. Extending the result of Theorem 4.7 to discontinuous processes seems to be a difficult task, see in particular Example 11.2.6 in [5]. One may, for example, obtain such an extension under the following condition which is not easy to verify in practice:

For every $\varepsilon>0$ there exists a strategy $H \in \mathscr{A}_{1}^{\mathbb{G}}$ with

$$
\mathbb{E}^{\mathbb{P}}\left[U\left(V_{T}^{1, H}\right)\right] \geq \sup _{H \in \mathscr{A}_{1}^{\mathbb{G}}} \mathbb{E}^{\mathbb{P}}\left[U\left(V_{T}^{1, H}\right)\right]-\varepsilon,
$$

and, for a number $n \geq 1$, a sequence of $\mathbb{G}^{n}$-predictable integrable processes $\left(H^{m}\right)$ as well as a sequence of $\mathbb{G}^{n}$-stopping times $\left(\tau_{m}\right)$ with $\mathbb{P}\left[\tau_{m}=T\right] \rightarrow 1$, such that for every $m \geq 1$,

$$
\left|\int_{0}^{t}\left(H_{t}^{m}-H_{t}\right) d S_{t}\right| \leq \varepsilon
$$

for all $t \leq \tau_{m}$

As a corollary to Theorems 4.7 and 3.8, we obtain a dual representation for the utility maximization problem in the case of non-atomic $G$.

Theorem 4.9. Let $S$ be a continuous semimartingale, suppose that Assumptions 2.6 and 4.5 hold true, and assume that

(i) The function $U:(0, \infty) \rightarrow \mathbb{R}$ is strictly concave, increasing, continuously differentiable and satisfies the Inada conditions at 0 and $\infty$.

(ii) For every $y \in(0, \infty)$, there exists $Z \in \operatorname{ELMMD}(\mathbb{F}, \mathbb{P})$ with $\mathbb{E}^{\mathbb{P}}[V(y Z)]<\infty$, where $V(y)=\sup _{x}(U(x)-x y)$.

Then,

$$
\sup _{H \in \mathscr{A}_{1}^{\mathbb{G}}} \mathbb{E}^{\mathbb{P}}\left[U\left(V_{T}^{1, H}\right)\right]=\lim _{n \rightarrow \infty} \sum_{i} \inf _{y>0}\left\{y+\inf _{Z \in E L M M D(\mathbb{F}, \mathbb{P})} \mathbb{E}^{\mathbb{P}}\left[V\left(y Z_{T}\right) 1_{G \in \Gamma_{i}^{n}}\right]\right\} .
$$

In the case of logarithmic utility, a more explicit expression may be obtained.

Corollary 4.10. Let $S$ be a continuous semimartingale, suppose that Assumptions 2.6 and 4.5 hold true, and assume that $G$ has a continuous density $f(x)$ and a finite entropy and that there exists $Z \in E L M M D(\mathbb{F}, \mathbb{P})$ with $\mathbb{E}^{\mathbb{P}}\left[\log Z_{T}\right]>-\infty$. Then the insider's expected log-utility is

$$
\begin{aligned}
\sup _{H \in \mathscr{A}_{1}^{\mathbb{G}}} \mathbb{E}^{\mathbb{P}}\left[\log V_{T}^{1, H}\right] & =-\int f(x) \log f(x) d x \\
& +\lim _{n \rightarrow \infty} \sum_{i=1}^{n}\left(-\log \left|\Gamma_{i}^{n}\right| \mathbb{P}\left[G \in \Gamma_{i}^{n}\right]+\inf _{Z \in E L M M D(\mathbb{F}, \mathbb{P})} \mathbb{E}^{\mathbb{P}}\left[1_{G \in \Gamma_{i}^{n}} \log \frac{1}{Z_{T}}\right]\right) .
\end{aligned}
$$


Proof. Theorem 4.7 and Corollary 3.10 show that

$$
\sup _{H \in \mathscr{A}_{1}^{\mathbb{G}}} \mathbb{E}^{\mathbb{P}}\left[\log V_{T}^{1, H}\right]=\lim _{n \rightarrow \infty} \sum_{i=1}^{n}\left(-\mathbb{P}\left[G \in \Gamma_{i}^{n}\right] \log \mathbb{P}\left[G \in \Gamma_{i}^{n}\right]+\inf _{Z \in E L M M D(\mathbb{F}, \mathbb{P})} \mathbb{E}^{\mathbb{P}}\left[1_{G \in \Gamma_{i}^{n}} \log \frac{1}{Z_{T}}\right]\right)
$$

Now we consider the first term in the right hand side. Using the mean value theorem, we have that $\mathbb{P}\left[G \in \Gamma_{i}^{n}\right]=f\left(x_{i}^{n}\right)\left|\Gamma_{i}^{n}\right|$ for some $x_{i}^{n} \in \Gamma_{i}^{n}$. Thus,

$$
\begin{aligned}
-\mathbb{P}\left[G \in \Gamma_{i}^{n}\right] \log \mathbb{P}\left[G \in \Gamma_{i}^{n}\right] & =-\mathbb{P}\left[G \in \Gamma_{i}^{n}\right] \log \left(f\left(x_{i}^{n}\right)\left|\Gamma_{i}^{n}\right|\right) \\
& =-f\left(x_{i}^{n}\right) \log f\left(x_{i}^{n}\right)\left|\Gamma_{i}^{n}\right|-\mathbb{P}\left[G \in \Gamma_{i}^{n}\right] \log \left|\Gamma_{i}^{n}\right| .
\end{aligned}
$$

Letting $n$ tend to infinity, we get the result.

As a consequence, the insider's log-utility problem is finite if $G$ has finite entropy and for every event $\left\{G \in \Gamma_{i}^{n}\right\}$, there exists a martingale density $Z_{T}$ such that the quantity $\mathbb{E}^{\mathbb{P}}\left[1_{G \in \Gamma_{i}^{n}} \log \left(1 / Z_{T}\right)\right]$ can compensate the term $-\log \left|\Gamma_{i}^{n}\right| \mathbb{P}\left[G \in \Gamma_{i}^{n}\right]$. In complete markets, it is impossible to find such a martingale density for each event, implying that expected log-utility of the insider is infinite. In incomplete markets, the result provides us with a new criterion for NUPBR under $\mathbb{G}$ as stated in the following

Corollary 4.11. Under Assumption 2.9 if there exists a constant $C<\infty$ such that for all $a$ and all $\varepsilon>0$ small enough,

$$
\begin{aligned}
\sup _{Z \in E L M M D} \mathbb{E}\left[1_{G \in(a, a+\varepsilon)} \log Z_{T}\right] \geq & -\mathbb{P}[G \in(a, a+\varepsilon)] \log \mathbb{P}[G \in(a, a+\varepsilon)] \\
& -C \mathbb{P}[G \in(a, a+\varepsilon)]
\end{aligned}
$$

then the condition NUPBR holds under $\mathbb{G}$.

Proof. Consider (17) for partitions of the form $\Gamma_{i}^{n}=\left(a_{i}, a_{i}+\varepsilon_{n}\right)$ with $\varepsilon_{n} \downarrow 0$. Using then (18) in (17) one obtains

$$
\begin{aligned}
\sup _{H \in \mathscr{A}_{1}^{\mathbb{G}}} \mathbb{E}^{\mathbb{P}}\left[\log V_{T}^{1, H}\right] & =\lim _{n \rightarrow \infty} \sum_{i=1}^{n}\left(-\mathbb{P}\left[G \in \Gamma_{i}^{n}\right] \log \mathbb{P}\left[G \in \Gamma_{i}^{n}\right]+\inf _{Z \in E L M M D(\mathbb{F}, \mathbb{P})} \mathbb{E}^{\mathbb{P}}\left[1_{\left\{G \in \Gamma_{i}^{n}\right\}} \log \frac{1}{Z_{T}}\right]\right) \\
& \leq \lim _{n \rightarrow \infty} \sum_{i=1}^{n} C \mathbb{P}\left[G \in \Gamma_{i}^{n}\right]=C .
\end{aligned}
$$

The expected log-utility of the insider is bounded and hence, by Proposition 3.6, the condition NUPBR holds under $\mathbb{G}$.

\section{Compliance with Ethical Standards}

The authors declare that they do not have any conflicts of interest in relation to the present work.

\section{Appendix}

Lemma 4.12. Assume that $X, Y$ are two independent exponential random variables with parameters $\alpha, \beta$, respectively. Then the random variable $Z=\frac{\alpha X}{\beta Y}$ has density $1 /(1+z)^{2}$. 
Proof. For $z>0$. we compute the cumulative distribution of $Z$

$$
\begin{aligned}
\mathbb{P}[Z \leq z] & =\mathbb{P}\left[Y \geq \frac{\alpha X}{\beta z}\right]=\int_{0}^{\infty}\left(\int_{(\alpha x) /(\beta z)}^{\infty} \beta e^{-\beta y} d y\right) \alpha e^{-\alpha x} d x \\
& =\int_{0}^{\infty} e^{\frac{-\alpha x}{z}} \alpha e^{-\alpha x} d x=\frac{z}{1+z}
\end{aligned}
$$

The density of $Z$ is obtained by taking derivative of the cumulative distribution of $Z$ with respect to $z$.

Definition 4.13 (Optional projection - Definition 5.2.1 of [19]). Let X be a bounded (or positive) process, and $\mathbb{F}$ a given filtration. The optional projection of $X$ is the unique optional process ${ }^{\circ} \mathrm{X}$ which satisfies

$$
\mathbb{E}\left[X_{\tau} 1_{\tau<\infty}\right]={ }^{o} X_{\tau} 1_{\tau<\infty}
$$

almost surely for any $\mathbb{F}$-stopping time $\tau$.

The following result helps us to find the compensator of a process when passing to smaller filtrations.

Lemma 4.14. Let $\mathbb{G}, \mathbb{H}$ be filtrations such that $\mathscr{G}_{t} \subset \mathscr{H}_{t}$, for all $t \in[0, T]$. Suppose that the process $M_{t}:=X_{t}-\int_{0}^{t} \lambda_{u} d u$ is a $\mathbb{H}$-martingale, where $\lambda \geq 0$. Then the process $M_{t}^{G}:=X_{t}-\int_{0}^{t}{ }^{o} \lambda_{u} d u$ is a $\mathbb{G}$-martingale, where ${ }^{o} \lambda$ is the optional projection of $\lambda$ onto $\mathbb{G}$.

Proof. Since $\lambda_{u} \geq 0$, the optional projection ${ }^{\circ} \lambda$ exists and for fixed $u$, it holds that ${ }^{o} \lambda_{u}=\mathbb{E}\left[\lambda_{u} \mid \mathscr{G}_{u}\right]$ almost surely. If $0 \leq s<t$ and $H$ is bounded and $\mathscr{G}_{s}$-measurable, then, by Fubini's Theorem

$$
\begin{aligned}
\mathbb{E}\left[H\left(M_{t}^{G}-M_{s}^{G}\right)\right] & =\mathbb{E}\left[H\left(X_{t}-X_{s}\right)\right]-\int_{s}^{t} \mathbb{E}\left[H \mathbb{E}\left[\lambda_{u} \mid \mathscr{G}_{u}\right]\right] d u \\
& =\mathbb{E}\left[H\left(X_{t}-X_{s}\right)\right]-\int_{s}^{t} \mathbb{E}\left[H \lambda_{u}\right] d u \\
& =\mathbb{E}\left[H\left(M_{t}-M_{s}\right)\right]=0 .
\end{aligned}
$$

Hence $M^{G}$ is a $\mathbb{G}$-martingale.

\section{References}

[1] B. Acciaio, C. Fontana, And C. Kardaras, Arbitrage of the first kind and filtration enlargements in semimartingale financial models, Stochastic Processes and their Applications, 126 (2016), pp. 1761-1784. 
[2] A. Aks amit, T. Choulli, And M. Jeanblanc, On an optional semimartingale decomposition and the existence of a deflator in an enlarged filtration, in In Memoriam Marc Yor-Séminaire de Probabilités XLVII, Springer, 2015, pp. 187218.

[3] J. Amendinger, D. Becherer, And M. Schweizer, A monetary value for initial information in portfolio optimization, Finance and Stochastics, 7 (2003), pp. 29-46.

[4] J. Amendinger, P. Imkeller, And M. Schweizer, Additional logarithmic utility of an insider, Stochastic Processes and their Applications, 75 (1998), pp. 263-286.

[5] S. ANKIRCHNER, Information and semimartingales, $\mathrm{PhD}$ thesis, Humboldt Universität Berlin, 2005.

[6] S. ANkIRCHNER, S. DeREICH, AND P. IMKELleR, The Shannon information of filtrations and the additional logarithmic utility of insiders, The Annals of Probability, 34 (2006), pp. 743-778.

[7] S. ANKIRCHNER AND J. ZWIERZ, Initial enlargement of filtrations and entropy of Poisson compensators, Journal of Theoretical Probability, 24 (2011), pp. 93117.

[8] J. BALDEAUX AND E. Platen, Liability driven investments under a benchmark based approach. preprint, 2013.

[9] P. BRemaud, Point processes and queues: martingale dynamics, Springer, 1981.

[10] H. N. CHAU AND P. TANKOV, Market models with optimal arbitrage, SIAM Journal on Financial Mathematics, 6 (2015), pp. 66-85.

[11] N. H. CHAU, A Study of Arbitrage Opportunities in Financial Markets without Martingale Measures, PhD thesis, Università degli Studi di Padova / Université Paris Diderot - Paris 7, 2016.

[12] A. Danilova, M. Monoyios, And A. NG, Optimal investment with inside information and parameter uncertainty, Mathematics and Financial Economics, 3 (2010), pp. 13-38.

[13] F. Delbaen And W. SChAChERMAYER, A general version of the fundamental theorem of asset pricing, Mathematische Annalen, 300 (1994), pp. 463-520.

[14] D. Fernholz And I. Karatzas, On optimal arbitrage, The Annals of Applied Probability, 20 (2010), pp. 1179-1204.

[15] C. FONTANA, Weak and strong no-arbitrage conditions for continuous financial markets, International Journal of Theoretical and Applied Finance, 18 (2015), p. 1550005.

[16] A. Grorud AND M. Pontier, Insider trading in a continuous time market model, International Journal of Theoretical and Applied Finance, 1 (1998), pp. 331-347. 
[17] P. Imkeller, M. Pontier, AND F. Weisz, Free lunch and arbitrage possibilities in a financial market model with an insider, Stochastic Processes and their Applications, 92 (2001), pp. 103-130.

[18] J. JACOD, Grossissement initial, hypothèse $\left(H^{\prime}\right)$ et théorème de Girsanov, in Grossissements de filtrations: exemples et applications, Springer, 1985, pp. 1535 .

[19] M. Jeanblanc, M. Yor, And M. Chesney, Mathematical Methods for Financial Markets, Springer, 2009.

[20] T. Jeulin, Semi-Martingales et Grossissement d'une Filtration, vol. 833 of Lecture Notes in Mathematics, Springer, 1980.

[21] Y. KABANOV, C. KARDARAS, AND S. SONG, No arbitrage and local martingale deflators, arXiv preprint arXiv:1501.04363, (2015).

[22] Y. M. KABANOV, On the FTAP of Kreps-Delbaen-Schachermayer, in Statistics and Control of Random Processes. The Liptser Festschrift, World Scientific, 1997, pp. 191-203.

[23] Y. M. KABANOV AND D. O. KRAMKOV, Large financial markets: asymptotic arbitrage and contiguity, Theory of Probability and Its Applications, 39 (1994), pp. 182-187.

[24] I. KARATZAS AND C. KARDARAS, The numéraire portfolio in semimartingale financial models, Finance and Stochastics, 11 (2007), pp. 447-493.

[25] C. KARDARAS, Market viability via absence of arbitrage of the first kind, Finance and Stochastics, 16 (2012), pp. 651-667.

[26] C. Kardaras, D. Kreher, And A. Nikeghbali, Strict local martingales and bubbles, The Annals of Applied Probability, 25 (2015), pp. 1827-1867.

[27] A. Kohatsu-Higa And M. YamaZato, Insider Models with Finite Utility in Markets with Jumps, Applied Mathematics \& Optimization, 64 (2011), pp. 217 255.

[28] D. KRAmKov And W. SChaChermayer, The asymptotic elasticity of utility functions and optimal investment in incomplete markets, Annals of Applied Probability, (1999), pp. 904-950.

[29] S. LEVEnTAL AND A. V. SKOROHOD, A necessary and sufficient condition for absence of arbitrage with tame portfolios, The Annals of Applied Probability, (1995), pp. 906-925.

[30] P.-A. MEYER, Sur un théoreme de J. Jacod, Séminaire de probabilités de Strasbourg, 12 (1978), pp. 57-60.

[31] O. Mostovyi, Necessary and sufficient conditions in the problem of optimal investment with intermediate consumption, Finance and Stochastics, 19 (2015), pp. $135-159$.

[32] I. PIKovsky AND I. KARATZAS, Anticipative portfolio optimization, Advances in Applied Probability, 28 (1996), pp. 1095-1122. 
[33] P. PRotTER, Stochastic Integration and Differential Equations, Springer, 2nd ed., 2003.

[34] L. C. G. Rogers AND D. Williams, Diffusions, Markov processes and martingales, volume I, 1979.

[35] J. RUF AND W. RUNGGALDIER, A systematic approach to constructing market models with arbitrage, Arbitrage, Credit and Informational Risks. Proceedings of the Sino-French Research Program in Financial Mathematics Conference, Beijing, (2013).

[36] S. Song, An alternative proof of a result of Takaoka, arXiv:1306.1062, (2013).

[37] K. TAKAOKA AND M. SCHWEIZER, A note on the condition of no unbounded profit with bounded risk, Finance and Stochastics, 18 (2014), pp. 393-405. 